\title{
Residue formulas for prepotentials, instanton expansions and conformal blocks
}

\author{
P. Gavrylenko ${ }^{a, b, c}$ and A. Marshakov ${ }^{d, e, a}$ \\ ${ }^{a}$ Department of Mathematics and Laboratory of Mathematical Physics, NRU HSE, \\ Vavilova 7, Moscow, Russia \\ ${ }^{b}$ Bogolyubov Institute for Theoretical Physics, \\ Metrologichna 14-b, Kiev, Ukraine \\ ${ }^{c}$ Department of Physics, Kyiv National University, \\ Glushkova 2, Kiev, Ukraine \\ ${ }^{d}$ Theory Department, Lebedev Physics Institute, \\ Leninskiy Prospekt 53, Moscow, Russia \\ ${ }^{e}$ Institute for Theoretical and Experimental Physics, \\ Bolshaya Cheryomushkinskaya 25, Moscow, Russia \\ E-mail: pasha145@gmail.com, mars@itep.ru
}

ABSTRACT: We study the extended prepotentials for the S-duality class of quiver gauge theories, considering them as quasiclassical tau-functions, depending on gauge theory condensates and bare couplings. The residue formulas for the third derivatives of extended prepotentials are proven, which lead to effective way of their computation, as expansion in the weak-coupling regime. We discuss also the differential equations, following from the residue formulas, including the WDVV equations, proven to be valid for the $\mathrm{SU}(2)$ quiver gauge theories. As a particular example we consider the constrained conformal quiver gauge theory, corresponding to the Zamolodchikov conformal blocks by $4 \mathrm{~d} / 2 \mathrm{~d}$ duality. In this case part of the found differential equations turn into nontrivial relations for the period matrices of hyperelliptic curves.

Keywords: Integrable Hierarchies, Supersymmetric Effective Theories, Integrable Equations in Physics, Conformal and W Symmetry

ARXIV EPRINT: 1312.6382 


\section{Contents}

1 Introduction 1

2 Generalities 3

2.1 Integrability 3

2.2 Residue formula 6

2.3 AGT-correspondence and residue formulas 8

3 Weak-coupling expansions of the prepotentials $\quad 9$

$\begin{array}{ll}3.1 \text { Methods for the weak-coupling expansion } & 10\end{array}$

3.2 Warm-up examples 11

$\begin{array}{ll}3.3 & \text { Quiver gauge theory and S-duality class } \\ \end{array}$

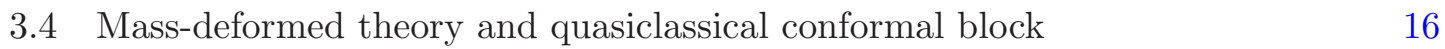

4 Zamolodchikov's conformal blocks $\quad 19$

5 Non-linear equations in quiver gauge theory 22

5.1 Relations for the period matrix 23

$\begin{array}{ll}\text { 5.2 WDVV equations from residue formula } & 25\end{array}$

5.3 Proof for the quiver gauge theory 26

$\begin{array}{llr}6 & \text { Conclusion } & 27\end{array}$

A Conformal block in the Ashkin-Teller model 28

B More on derivatives of the period matrices 30

\section{Introduction}

The S-duality class of the supersymmetric quiver theories [1] allows to study gauge theories with matter, charged with respect to more than a single gauge group. In some regions of their moduli space the traditional methods of quantum field theory are not applicable, but they can be still analyzed, using geometric approach to $\mathcal{N}=2$ supersymmetric gauge theories, initiated long ago by Seiberg and Witten [2]. It is especially intriguing and interesting, that this geometry can be independently viewed both from four-dimensional and two-dimensional perspectives [3-7], allowing to apply in the latter case the dual techniques of theories with infinite-dimensional algebras of symmetry. Direct observation of such symmetry in four-dimensional gauge theories remains to be one of the main challenges in modern mathematical physics.

The Seiberg-Witten (SW) prepotentials in quiver gauge theories can be naturally extended [8] to incorporate the dependence of the bare ultraviolet (UV) couplings in addition to the infrared (IR) gauge theory condensates. In this way they can be identified with more 
general class of the quasiclassical tau-functions [9], which are well-known from long ago [10] to appear in the context of supersymmetric gauge theories. Studied previously only for the higher perturbations of the UV prepotential [3, 11-14], this extension becomes more generic for the quiver theories and can be studied in detail along the lines, proposed in [8].

One of the practical applications of the geometric picture and integrable equations in the gauge-theory framework is that it allows to use the alternative methods for the computations of the prepotentials, describing physics of light states in the IR. These methods equally work in the regions, where the theory possesses the UV non-Abelian Lagrangian description, as well as in the regions where only an effective description of light Abelian modes is possible (or even no Lagrangian is known at all [1]). In the first case the weak-coupling phase in $\mathcal{N}=2$ supersymmetric gauge theory is saturated by the oneloop perturbation theory and the instanton calculus $[2,15]$, while in the latter cases the traditional methods of quantum gauge theory are not fully applicable. Below we are going to use the techniques of the quasiclassical integrable systems [9] to study the expansion of the prepotentials of quiver gauge theories in various regions of the moduli space, to be called as weak-coupling expansion, since it coincides with the perturbative and instanton analysis in all known cases, though goes itself beyond the scope, where such analysis is valid.

In this paper the proposed methods will be used to study the prepotentials of S-duality class of the $\mathrm{SU}(2)$ quiver gauge theories, and mostly with the massless matter (vanishing bare masses). Already in this case there are some subtleties with the analysis of weaklycoupled phases with (half-) tri-fundamental multiplets (the so called sicilian quivers), where the instanton calculus [15] is not directly applicable [16]. Nevertheless, the developed methods lead directly to the weak-coupling expansion in this case as well, and this allows to hope on their validity for the most interesting case of the higher-rank gauge quivers, where the "sicilian problem" arises in full. Moreover, when one of the trifundamental states becomes massless, such theories form a class of four-dimensional conformal theories with the quadratic prepotentials, when the couplings are renormalized from their bare values only by non-perturbative effects (the perturbative beta-functions vanish and the perturbative renormalization is finite).

Fortunately, for this class of quiver theories there is a well-known description on twodimensional side, proposed by Al. Zamolodchikov in terms of conformal blocks for the $c=1$ Ashkin-Teller (AT) model or scalar field on hyperelliptic Riemann surface [18]. In the SW approach this Riemann surface appears just as a particular degenerate case of the SW curve for a massless $\mathrm{SU}(2)$ quiver, when the gauge theory condensates are constrained by certain conservation condition. We establish here direct relation of the geometric approach with the formulation in terms of two-dimensional conformal field theory (2d CFT), which is one more nice example of $4 \mathrm{~d} / 2 \mathrm{~d}$ correspondence, going — strictly speaking - even beyond the framework of the AGT conjecture [7], since generic Nekrasov function is now known in such cases [16] (see also [17]).

We derive a generalization of the Zamolodchikov renormalization formula [19-21] (see also [8, 22-25]) for this class of constrained quiver theories, which includes the first-order differential equations for the effective couplings and their implicit solution via the Thomae formulas. Finally, we study another kind of non-linear differential equations for the ex- 
tended prepotentials - the WDVV equations [26-29], directly following from the residue formulas, and show that they hold both in generic massless and constrained Zamolodchikov's cases.

This paper is organized as follows. Section 2 contains the review of the SW approach, and its extension to the tau-functions of the quiver gauge theories. We also demonstrate here, that part of the formulas can be immediately derived, using the AGT correspondence with conformal theory, and prove existence of the standard residue formula [9] for the third derivatives of the extended quiver prepotentials.

In section 3 we propose two slightly different, but directly related methods of calculating the weak-coupling expansions of the prepotentials, based on differential equations, arising from the residue formulas and the period integral expansions. We reproduce in this way few well-known examples, and study in detail two quiver gauge models (including the case of sicilian quiver in section 3.3) and massive deformations of conformal gauge theories. Section 4 contains the analysis of connection between the constrained quiver theories and exact Zamolodchikov's conformal blocks in AT model, we prove that the SW description coincides with the 2 d CFT result. Section 5 contain the discussion of the nonlinear differential equations for the quiver tau-functions. We show, that the simplest relations for the period matrices of Zamolodchikov's case are equivalent to the well-known Rauch formulas, which describe here the nonperturbative renormalization of the UV couplings in conformal theories. This section also contains the proof that the prepotential solves the WDVV equations [29] as the function of all variables: both in the full massless theory, and in the constrained case. Section 6 is devoted to brief discussion of our results. Some technical information is contained in appendices.

\section{Generalities}

\subsection{Integrability}

We start from the definition of the SW system [2], assuming here the S-duality class of quiver gauge theories [1] with the $\bigotimes_{k} \mathrm{SU}\left(N_{c}^{(k)}\right)$ gauge groups. We shall mostly concentrate on the superconformal models, containing fundamental, bifundamental and trifundamental matter multiplets in a combination, which gives vanishing $\beta$-functions $\beta_{k}=0$ for all simple gauge group components, moreover — often with vanishing bare masses. The asymptotically free cases can be obtained from superconformal models with dimensionless bare couplings by standard dynamical transmutation in the limit when (some of) the bare masses become infinite and corresponding multiplets decouple.

The definition includes:

- $g$-parametric family of the genus $g$ curves $\Sigma: F\left(x, z ; u_{1}, \ldots, u_{g}\right)=0$ with the fixed basis in $H_{1}(\Sigma, \mathbb{Z})$ (including marked $A$ - and $B$-cycles).

- Two meromorphic differentials $d x$ and $d z$ or the SW one-form $d S=x d z$.

- The connection $\nabla$ on the moduli space, such that derivatives $\nabla_{\frac{\partial}{\partial u_{i}}} d S$ are holomorphic. 
The SW equations read (see [9] for the most general construction and [2] for the first application to supersymmetric gauge theory)

$$
a_{i}=\frac{1}{2 \pi i} \oint_{A_{i}} d S, \quad a_{i}^{D}=\oint_{B_{i}} d S=\frac{\partial \mathcal{F}}{\partial a_{i}} \quad i=1, \ldots, g .
$$

Theorem 1. There exists locally-defined function $\mathcal{F}\left(a_{1}, \ldots a_{g}\right)$, which solves this system, the difference of any two solutions is a-independent.

Proof. Denote $\nabla_{\frac{\partial}{\partial a_{i}}}=\frac{\partial}{\partial a_{i}}$ and compute the derivatives

$$
\delta_{i j}=\frac{1}{2 \pi i} \oint_{A_{i}} \frac{\partial d S}{\partial a_{j}} \quad \frac{\partial a_{i}^{D}}{\partial a_{j}}=\oint_{B_{i}} \frac{\partial d S}{\partial a_{j}}=\mathcal{T}_{i j} .
$$

We conclude from the first formula that $\frac{\partial d S}{\partial a_{i}}=d \omega_{i}, i=1, \ldots, g$ are canonically-normalized holomorphic one-forms, so the second derivatives of the prepotential are the period matrix, which is symmetric due to Riemann bilinear relations (RBR)

$$
\begin{aligned}
0=\int_{\Sigma} d \omega_{i} \wedge d \omega_{j}=\int_{\partial \Sigma_{\mathrm{cut}}} \omega_{i} d \omega_{j} & =\sum_{k=1}^{g}\left(\oint_{A_{k}} d \omega_{i} \oint_{B_{k}} d \omega_{j}-\oint_{A_{k}} d \omega_{j} \oint_{B_{k}} d \omega_{i}\right)= \\
& =\mathcal{T}_{i j}-\mathcal{T}_{j i}
\end{aligned}
$$

where we have used the Stokes formula for the integral over the boundary $\partial \Sigma_{\text {cut }}$ of the cut Riemann surface $\Sigma$. Hence, we have proven that 1-form on the moduli space $\eta=$ $\sum_{i=1}^{g} \delta a_{i} a_{i}^{D}=\sum_{i=1}^{g} \delta\left(\oint_{A_{i}} d S\right) \cdot \oint_{B_{i}} d S$ is closed, so locally it is the differential of some function $\mathcal{F}$.

Now let us extend and precise our definition, for the family of curves

$$
F(x, z ; \mathbf{u}, \mathbf{q})=F\left(x, z ; u_{1}, \ldots, u_{g} ; q_{1}, \ldots, q_{n}\right)=\sum_{k} x^{k} \phi_{k}(z)=0
$$

which covers $\Sigma \rightarrow \Sigma_{0}$ some curve $\Sigma_{0}$ (which is often called UV or Gaiotto curve), whose moduli space can be parameterized by $\mathbf{q}=\left\{q_{1}, \ldots, q_{n}\right\}$, while $\mathbf{u}=\left\{u_{1}, \ldots, u_{g}\right\}$ are moduli of the cover. ${ }^{1}$ The curve $\Sigma$ is endowed with two meromorphic differentials [9]: $d z$, which

\footnotetext{
${ }^{1}$ In the most simple, but still important case the cover is hyperelliptic, defined by the quadratic equation $x^{2}=\phi_{2}(z)$, with the poles at $n$ marked points. In this case the number of vacuum condensates (the dimension of the space of such differentials) equals to the dimension of Teichmüller space of $\Sigma_{0}: l\left(2 K+p_{1}+\right.$ $\left.\ldots+p_{n}\right)=4 g_{0}-4+n-g_{0}+1=3 g_{0}-3+n=\operatorname{dim}$ Teich $_{g_{0}, n}$, i.e. the number of coupling constants equals to the number of vacuum condensates (each $\mathrm{SU}(2)$ gauge group comes with the coupling constant and the vacuum condensate), and the whole set of parameters can be identified with $T^{*}\left(\operatorname{Teich}_{g_{0}, n}\right)$. The genus of the cover $\Sigma$ comes from the Riemann-Hurwiz formula: $g=2 g_{0}-1+\frac{1}{2} \sum\left(n_{i}-1\right)=2 g_{0}-1+2 g_{0}-2+n=g_{0}+\left(3 g_{0}-3+n\right)$. Unfortunately, only partially such analysis can be applied to the case of higher-rank gauge quivers, see [8]. However, we shall also consider below the Zamolodchikov or constrained case, where the number of gauge theory condensates is constrained by certain conservation conditions (or vanishing of the masses of some light physical states), but the number of UV couplings remains intact, then the reduced genus $\tilde{g}<n$.
} 
can be projected to the UV curve $\Sigma_{0}$ and $d x$, or generating one-form $d S=x d z$. The connection $\nabla=\nabla^{z}$ can be now defined via projection onto the $z$-plain:

$$
\nabla_{\frac{\partial}{\partial u_{i}}} f(z, x(z, \mathbf{u})) d z=\frac{\partial f(z, x(z, \mathbf{u})}{\partial u_{i}} d z .
$$

For the rational $\Sigma_{0}$ with $g_{0}=0$ parameters $\left\{q_{i}\right\}$ are the co-ordinates of the following points in $z$-plane (both options are possible):

- the branch-points of the cover, where $x \underset{z \rightarrow q_{i}}{=} \sum_{l=1}^{k-1} C_{l}(z, \mathbf{q}, \vec{u})\left(z-q_{i}\right)^{-\frac{l}{k}}$ (massless case).

- The set of the regular points on the cover $\Sigma$, where $x d z$ has the simple poles with fixed residues (massive case).

Consider now the following system of equations:

$$
\frac{\partial \mathcal{F}}{\partial q_{i}}=\frac{1}{2} \sum_{p^{-1}\left(q_{i}\right)} \operatorname{Res} \frac{(d S)^{2}}{d z}, \quad i=1, \ldots, n .
$$

Here we should compute the number of points with their multiplicities. Then there is the non-trivial

Theorem 2. The systems (2.1) and (2.4) are consistent. They define $\mathcal{F}(\mathbf{a}, \mathbf{q})$, which is defined up to some constant, whereas (2.1) defines $\mathcal{F}(\mathbf{a}, \mathbf{q})$ up to some $q_{i}$-dependent function.

Proof. In the vicinity of branching points one has

$$
\begin{aligned}
& d S^{(1)} \underset{z \rightarrow q_{i}}{=} \sum_{l=1}^{k-1} \frac{C_{l}(z, \mathbf{q}, \mathbf{a}) d z}{\left(z-q_{i}\right)^{\frac{l}{k}}} \\
& d S^{(2)} \underset{z \rightarrow q_{i}}{=} \frac{m_{i} d z}{z-q_{i}}+C_{0}(z, \mathbf{q}, \mathbf{a}) d z
\end{aligned}
$$

where two different possibilities are marked by ${ }^{(1)}$ or ${ }^{(2)}$ respectively. Here $C_{l}(z, \mathbf{q}, \mathbf{a})=$ $C_{l}+c_{1 l}\left(z-q_{i}\right)+c_{2 l}\left(z-q_{i}\right)^{2}+\ldots$ are analytic at $z \rightarrow q_{i}$, therefore

$$
\begin{aligned}
& \frac{\partial d S^{(1)}}{\partial q_{i}} \underset{z \rightarrow q_{i}}{=} \sum_{l=1}^{k-1} \frac{l}{k} \frac{C_{l} d z}{\left(z-q_{i}\right)^{\frac{l}{k}+1}}+\text { hol. } \underset{z \rightarrow q_{i}}{=}-d \sum_{l=1}^{k} \frac{C_{l} d z}{\left(z-q_{i}\right)^{\frac{l}{k}}}+\text { hol. } \\
& \frac{\partial d S^{(2)}}{\partial q_{i}} \underset{z \rightarrow q_{i}}{=} \frac{m_{i} d z}{\left(z-q_{i}\right)^{2}}+\text { hol. } \underset{z \rightarrow q_{i}}{=}-d \frac{m_{i}}{z-q_{i}}+\text { hol. }
\end{aligned}
$$

since the residues are fixed, or $\frac{\partial m_{i}}{\partial q_{i}}=0$. Denote $\frac{\partial d S}{\partial q_{i}}=d \Omega_{i}$, then in both cases

$$
\frac{d S}{d z} \underset{z \rightarrow q_{i}}{=}-\Omega_{i}+\text { hol }
$$


where $\Omega_{i}(P)=\int_{P_{0}}^{P} d \Omega_{i}$ is corresponding Abelian integral. For the mixed derivatives one gets from (2.4)

$$
\frac{\partial \mathcal{F}}{\partial a_{j} \partial q_{i}}=\sum_{p^{-1}\left(q_{i}\right)} \operatorname{Res} \frac{d S}{d z} \frac{\partial d S}{\partial a_{j}}=\sum_{p^{-1}\left(q_{i}\right)} \operatorname{Res} \frac{d S}{d z} d \omega_{j}=-\sum \operatorname{Res}\left(\Omega_{i} d \omega_{j}\right)
$$

where the replacement $\frac{d S}{d z} \underset{z \rightarrow q_{i}}{\approx}-\Omega_{i}$ is allowed, because $d \omega_{j}$ is holomorphic. On the other hand from (2.1)

$$
\begin{aligned}
\frac{\partial^{2} \mathcal{F}}{\partial q_{i} \partial a_{j}} & =\oint_{B_{j}} \frac{\partial d S}{\partial q_{i}}=\oint_{B_{j}} d \Omega_{i}=\frac{1}{2 \pi i} \sum_{l=1}^{g}\left(\oint_{A_{l}} d \omega_{j} \oint_{B_{l}} d \Omega_{i}-\oint_{B_{l}} d \omega_{j} \oint_{A_{l}} d \Omega_{i}\right)= \\
& =-\frac{1}{2 \pi i} \oint_{\partial \Sigma_{\mathrm{cut}}} \Omega_{i} d \omega_{j}=-\sum \operatorname{Res}\left(\Omega_{i} d \omega_{j}\right)
\end{aligned}
$$

where we have used $0=\frac{\partial a_{k}}{\partial q_{i}}=\oint_{A_{k}} d \Omega_{j}$, and we sum over all poles of $\Omega_{i}$. So we have proven that $\frac{\partial^{2} \mathcal{F}}{\partial a_{j} \partial q_{i}}=\frac{\partial^{2} \mathcal{F}}{\partial q_{i} \partial a_{j}}$, which means the consistency of equations.

Now consider the second set of the mixed derivatives

$$
\frac{\partial^{2} \mathcal{F}}{\partial q_{j} \partial q_{i}}=\sum_{p^{-1}\left(q_{i}\right)} \operatorname{Res} \frac{d S}{d z} d \Omega_{j}=-\sum_{p^{-1}\left(q_{i}\right)} \operatorname{Res}\left(\Omega_{i} d \Omega_{j}\right)
$$

which gives for the difference

$$
\begin{aligned}
\frac{\partial^{2} \mathcal{F}}{\partial q_{j} \partial q_{i}}-\frac{\partial^{2} \mathcal{F}}{\partial q_{i} \partial q_{j}} & =-\sum_{p^{-1}\left(q_{i}\right)} \operatorname{Res}\left(\Omega_{i} d \Omega_{j}\right)+\sum_{p^{-1}\left(q_{j}\right)} \operatorname{Res}\left(\Omega_{j} d \Omega_{i}\right)= \\
& =-\sum_{p^{-1}\left(q_{i}\right)} \operatorname{Res} d\left(\Omega_{i} \Omega_{j}\right)+\sum \operatorname{Res}\left(\Omega_{j} d \Omega_{i}\right) .
\end{aligned}
$$

Here the first term is zero due to the trivial reason, and second is zero due to

$$
\sum \operatorname{Res}\left(\Omega_{j} d \Omega_{i}\right)=\frac{1}{2 \pi i} \sum_{l=1}^{g}\left(\oint_{A_{l}} d \Omega_{j} \oint_{B_{l}} d \Omega_{i}-\oint_{B_{l}} d \Omega_{j} \oint_{A_{l}} d \Omega_{i}\right)=0 .
$$

Hence, all mixed second derivatives are equal due to the RBR.

\subsection{Residue formula}

The third derivatives of quasiclassical tau-functions should be generally presented by the elegant residue formulas [9]. To prove it for our case we unify all variables into a single set $\left\{X_{I}\right\}=\left\{a_{i}\right\} \cup\left\{q_{k}\right\}$, and the same with the differentials: $\left\{d \varpi_{I}\right\}=\left\{d \omega_{i}\right\} \cup\left\{d \Omega_{k}\right\}$.

Theorem 3. There is a set of residue formulas for the third derivatives of the generalized prepotential defined by (2.1) and (2.4)

$$
\frac{\partial^{3} \mathcal{F}}{\partial X_{I} \partial X_{J} \partial X_{K}}=\sum_{d x=0} \operatorname{Res} \frac{d \varpi_{I} d \varpi_{J} d \varpi_{K}}{d x d z} .
$$


Proof. Let us consider the most subtle case of the third q-derivatives

$$
\frac{\partial^{3} \mathcal{F}}{\partial q_{i} \partial q_{j} \partial q_{k}}=\sum_{d x=0} \operatorname{Res} \frac{d \Omega_{i} d \Omega_{j} d \Omega_{k}}{d x d z} .
$$

Formulas for the third a-derivatives (see, e.g. [30]) and mixed derivatives are proven just in the same way.

Start with

$$
\frac{\partial^{2} \mathcal{F}}{\partial q_{j} \partial q_{i}}=\sum_{p^{-1}\left(q_{i}\right)} \operatorname{Res} \frac{d S}{d z} d \Omega_{j}=\sum_{p^{-1}\left(q_{i}\right)} \operatorname{Res} x d \Omega_{j} .
$$

To take extra $q$-derivative it is more convenient to use connection $\nabla^{x}$, which is defined via the projection onto the $x$-plane:

$$
\nabla_{\frac{\partial}{\partial q_{i}}}^{x} d \Omega_{j}=\frac{\partial}{\partial q_{i}} d \Omega_{j}(x, z(x, \mathbf{u}))=\left.\frac{\partial}{\partial q_{i}} d \Omega_{j}\right|_{x}
$$

so that

$$
\begin{aligned}
\frac{\partial^{3} \mathcal{F}}{\partial q_{j} \partial q_{i} \partial q_{k}} & =\left.\sum_{p^{-1}\left(q_{i}\right)} \operatorname{Res} x \frac{\partial d \Omega_{j}}{\partial q_{k}}\right|_{x}=-\left.\sum_{p^{-1}\left(q_{i}\right)} \operatorname{Res} \Omega_{i} \frac{\partial d \Omega_{j}}{\partial q_{k}}\right|_{x}= \\
& =\left.\sum_{p^{-1}\left(q_{i}\right)} \operatorname{Res} d \Omega_{i} \frac{\partial \Omega_{j}}{\partial q_{k}}\right|_{x}=\left.\frac{1}{2 \pi i} \oint_{\partial \Sigma_{\mathrm{cut}}} d \Omega_{i} \frac{\partial \Omega_{j}}{\partial q_{k}}\right|_{x}-\left.\sum_{d x=0} \operatorname{Res} d \Omega_{i} \frac{\partial \Omega_{j}}{\partial q_{k}}\right|_{x}= \\
& =-\left.\sum_{d x=0} \operatorname{Res} d \Omega_{i} \frac{\partial \Omega_{j}}{\partial q_{k}}\right|_{x}
\end{aligned}
$$

where we have used the fact, that singular part of $d \Omega_{j}$ near $q_{i}$ is proportional to $d x$, so the derivative $\left.\frac{\partial d \Omega_{j}}{\partial q_{k}}\right|_{x}$ is holomorphic, and transformed expression into the sum over all branch points $d x=0$ using the integral over the border of the cut $\Sigma$ and normalization $\oint_{A_{i}} d \Omega_{j}=0$.

Now the sum goes over the branch-points of the $x$-projection, we assume without loss of generality, that these ramification points are simple. In the vicinity of each such point with $(z, x)=\left(z^{*}, x^{*}\right)$ one can write (up to the terms, not giving contribution to the final formula, which is denoted by " $\approx$ ")

$$
x \approx x^{*}+a\left(z-z^{*}\right)^{2}, \quad z \approx z^{*}+\sqrt{\frac{x-x^{*}}{a}}, \quad d z \approx \frac{d x}{2 \sqrt{a\left(x-x^{*}\right)}}
$$

then

$$
\begin{array}{rlrl}
d S & \approx x^{*} d z, & d \Omega_{k}=\left.\frac{\partial d S}{\partial q_{k}}\right|_{z} \approx \frac{\partial x^{*}}{\partial q_{k}} d z \\
\Omega_{k} \approx \frac{\partial x^{*}}{\partial q_{k}} z \approx \frac{\partial x^{*}}{\partial q_{k}} \sqrt{\frac{x-x^{*}}{a}}, & \left.\frac{\partial \Omega_{j}}{\partial q_{k}}\right|_{x} \approx-\frac{\frac{\partial x^{*}}{\partial q_{j}} \frac{\partial x^{*}}{\partial q_{k}}}{2 \sqrt{a\left(x-x^{*}\right)}}
\end{array}
$$

and therefore

$$
\frac{\partial x^{*}}{\partial q_{k}} \approx \frac{d \Omega_{k}}{d z},\left.\quad \frac{\partial \Omega_{j}}{\partial q_{k}}\right|_{x} \approx-\frac{d \Omega_{j}}{d z} \frac{d \Omega_{k}}{d z} \frac{d z}{d x}=-\frac{d \Omega_{j} d \Omega_{k}}{d x d z}
$$

so that, substituting into (2.17), we finally get (2.14). 
Note, that this formula is proven here almost in a full generality, therefore it will be used below in all cases we need.

\subsection{AGT-correspondence and residue formulas}

The spectral curve of the large S-duality class of the quiver theories [1] can be written in the form of (2.3), where the $k$-differentials $\left\{\phi_{k}\right\}$ are defined on the UV-curve $\Sigma_{0}$ - in many cases just on Riemann sphere with marked points $\left\{z_{i}\right\}=\left\{0,1, \infty, q_{1}, q_{2}, q_{3}, \ldots\right\}$, where they are allowed to have some prescribed singularities. The positions of these singularities $\left\{q_{i}\right\}$ parameterize the space of UV coupling-constants in the theory.

The picture is very simple in the case of $\mathrm{SU}(2)$-quiver theory, where it has clear interpretation in terms of two-dimensional CFT. The spectral curve equation (2.3)

$$
x^{2}=\phi_{2}(z)=\langle T(z)\rangle=\sum_{j=1}^{n}\left(\frac{\Delta_{j}}{\left(z-z_{j}\right)^{2}}+\frac{u_{j}}{z-z_{j}}\right)
$$

leads immediately to the residue formulas (2.4) for the first derivatives of prepotentials in terms of the generating differential $d S=x d z$

$$
u_{i}=\operatorname{res}_{z=z_{i}} x^{2} d z=\operatorname{res}_{z=z_{i}} \frac{d S d S}{d z}=\frac{1}{2} \operatorname{Res}_{P_{i}^{ \pm}} \frac{d S d S}{d z}=\frac{\partial \mathcal{F}}{\partial z_{i}} .
$$

Taking one more derivative (at constant $z$ ) one gets

$$
2 x \frac{\partial x}{\partial z_{j}}=\frac{2 \Delta_{j}}{\left(z-z_{j}\right)^{3}}+\frac{u_{j}}{\left(z-z_{j}\right)^{2}}+\frac{\partial u_{j} / \partial z_{j}}{z-z_{j}}+\sum_{i \neq j} \frac{\partial u_{i} / \partial z_{j}}{z-z_{i}}
$$

that is

$$
\begin{aligned}
\frac{\partial^{2} \mathcal{F}}{\partial z_{j}} & =2 \operatorname{res}_{z=z_{j}} x \frac{\partial x}{\partial z_{j}} d z=2 \operatorname{res}_{z=z_{j}} \frac{d S d \Omega_{j}}{d z} \\
\frac{\partial^{2} \mathcal{F}}{\partial z_{i} \partial z_{j}} & =2 \operatorname{res}_{z=z_{i}} x \frac{\partial x}{\partial z_{j}} d z=2 \operatorname{res}_{z=z_{i}} \frac{d S d \Omega_{j}}{d z}, \quad i \neq j
\end{aligned}
$$

where

$$
d \Omega_{j}=\frac{\partial x}{\partial z_{j}} d z=\nabla_{\frac{\partial}{\partial z_{i}}}^{z} x d z=\nabla_{\frac{\partial}{\partial z_{i}}}^{z} d S
$$

and the derivatives

$$
\frac{\partial u_{k}}{\partial z_{i}}=\left.\frac{\partial u_{k}}{\partial z_{i}}\right|_{\mathbf{a}}
$$

are taken at constant a-periods $\mathbf{a}=\frac{1}{2 \pi i} \oint_{\mathbf{A}} x d z$ of the generating differential or some fixed choice of the cycles $\left\{A_{j}\right\}$ on the cover.

Notice, that these formulas are not all independent due to constraints, coming from the regularity condition at $z=\infty$

$$
\sum_{j=1}^{n} u_{j}=0, \quad \sum_{j=1}^{n}\left(z_{j} u_{j}+\Delta_{j}\right)=0, \quad \sum_{j=1}^{n}\left(z_{j}^{2} u_{j}+2 z_{j} \Delta_{j}\right)=0 .
$$


Consider now the reparameterization in the space of bare coupling induced by $z \rightarrow \omega(z)$, which can be conveniently encoded by $d \omega=d z / f(z)$. Then, the first derivatives of deformed prepotential $\mathcal{F}_{f}$ are given by

$$
f\left(z_{i}\right) \frac{\partial \mathcal{F}_{f}}{\partial z_{i}}=\operatorname{res}_{z=z_{i}} \frac{d S d S}{d z / f(z)}=\operatorname{res}_{z=z_{i}} f(z) x^{2} d z .
$$

Calculating the residue in the r.h.s. using (2.21) one finds, that

$$
f\left(z_{i}\right) \frac{\partial \mathcal{F}_{f}}{\partial z_{i}}=f\left(z_{i}\right) u_{i}+f^{\prime}\left(z_{i}\right) \Delta_{i}
$$

and this corresponds to the transformation

$$
\mathcal{F}_{f}=\mathcal{F}+\sum_{i=1}^{n} \Delta_{i} \log f\left(z_{i}\right)
$$

which does not change the derivatives of prepotential over the period a-variables.

Notice that the residue formula is also true for an arbitrary $d \omega=\frac{d z}{f(z)}$ :

$$
\frac{\partial^{3} \mathcal{F}_{f}}{\partial \omega\left(z_{i}\right) \partial \omega\left(z_{j}\right) \partial \omega\left(z_{k}\right)}=\sum_{d(x f(z))=0} \frac{d \Omega_{i}^{f} d \Omega_{j}^{f} d \Omega_{k}^{f}}{d(x f(z)) d \omega}
$$

where $d \Omega_{i}^{f}=\left.f\left(z_{i}\right) \frac{\partial d S}{\partial z_{i}}\right|_{z}$. It is clear, since the proof of section 2.2 can be rewritten literally for the differential $d z / f(z)$ and the function $x f(z)$.

Similarly one can consider the change of couplings, corresponding

$$
\left(z_{1}, \ldots, z_{n}\right) \rightarrow\left(q_{1}, \ldots, q_{n-3}, 1,0, \infty\right) .
$$

In particular, for $f(z)=\frac{\left(z-z_{n}\right)\left(z-z_{n-1}\right)}{z_{n}-z_{n-1}}$ with some fixed $\left(z_{n}, z_{n-1}, z_{n-2}\right)$ one gets

$$
\begin{aligned}
z_{j} \frac{\partial \mathcal{F}_{f}}{\partial z_{j}} & =\operatorname{res}_{z_{j}} \frac{d S}{d \omega} d S=\operatorname{res}_{z_{j}} \frac{x^{2} d z}{d \omega / d z}= \\
& =\frac{2 z_{j}-z_{n}-z_{n-1}}{z_{n}-z_{n-1}} \Delta_{j}+\frac{\left(z_{j}-z_{n}\right)\left(z_{j}-z_{n-1}\right)}{z_{n}-z_{n-1}} u_{j} \quad j=1, \ldots, n-3
\end{aligned}
$$

where $d \omega=\frac{d z}{f(z)}=\frac{d z}{z-z_{n}}-\frac{d z}{z-z_{n-1}}$. In this way one can easily reproduce all original formulas from $[8]$.

\section{Weak-coupling expansions of the prepotentials}

In this section we propose the techniques of the weak-coupling analysis of the quiver gauge theories, based on applications of the residue formulas. For the perturbative prepotentials - instead of computation of the period matrices of degenerate curves — one can just compute the residues of certain one-forms, which can be projected from the SW curve $\Sigma$ to the UV Gaiotto curve $\Sigma_{0}$. This procedure has been applied to the computations of the dependence of perturbative prepotentials over vacuum condensates in [31], and we extend it here to the tau-functions of quiver gauge theories as functions of the bare couplings. 
The dependence of perturbative prepotentials over the UV couplings is rather simple and can be directly compared with the one-loop calculations in corresponding supersymmetric quantum field theories. However, the application of our methods can be immediately extended to compute the whole weak-coupling expansion of a prepotential. ${ }^{2}$ One can apply for these purposes the differential equations, obtained from the residue formulas (2.14) for the third q-derivatives, and expressing them in terms of the first (2.4) and second (2.10) derivatives of the same prepotentials. Equivalently, one can compute the power corrections in bare couplings to the perturbative prepotentials by study of the q-expansions of the period integrals, which define the integration constants for these equations. These power corrections exactly correspond to the instanton expansions of the quiver gauge theories, but also go beyond this case, when the latter cannot be defined [16]. As an example, we compute the expansion for the case of sicilian quiver with three $\mathrm{SU}(2)$ groups, which will be also used later for discussion of the constrained or Zamolodchikov's case.

\subsection{Methods for the weak-coupling expansion}

An effective solution of the equations (2.1) is generally not so easy due to the complicated geometry of the spectral curve (see e.g. [34]). Fortunately, in the vicinity of particular points in the moduli space, where spectral curve degenerates, one can find the series expansions of the prepotential. Here we describe two different but closely related methods of such calculation.

\section{Method I:}

- parameterize a spectral curve through $\frac{\partial \mathcal{F}}{\partial q_{i}}$ using (2.4);

- substitute an expansion $\mathcal{F}=\mathbf{A} \log \mathbf{q}+\sum_{\mathbf{k}>0} c_{\mathbf{k}} \mathbf{q}^{\mathbf{k}}$ into the first half of equations in (2.1) and solve obtained equations iteratively;

- recover the q-independent part of the prepotential using (2.13) for the a-derivatives.

\section{Method II:}

- derive the non-linear differential equation for the prepotential as the function of $\mathbf{q}$ using (2.13), it expresses the third derivatives through the first (coefficients of the equation for the curve) and second (coefficients in the expressions for the differentials $d \Omega)$

- solve the first equation of (2.1) in the degenerate limit, and recover the term $\mathbf{A} \log \mathbf{q}$;

- substitute an expansion $\mathcal{F}=\mathbf{A} \log \mathbf{q}+\sum_{\mathbf{k}>0} c_{\mathbf{k}} \mathbf{q}^{\mathbf{k}}$ into obtained differential equation and solve it iteratively;

- recover again the q-independent part of the prepotential using (2.13) for the a-derivatives.

\footnotetext{
${ }^{2}$ The neighborhoods of degenerate curves in the moduli space are usually called as the weak-coupling regions, this is true indeed in many cases - the corresponding theory has a Lagrangian description, and the whole weak-coupling expansion can be recovered from the instanton calculus.
} 
Strictly speaking, the first method is just a modification of the second, since it uses directly the period integrals, which play the role of the integrals of motion for the differential equations we use in the second method of computation. In what follows we use both of them, dependently on particular example, to save the efforts.

\subsection{Warm-up examples}

Original SW theory. Let us start with the integrable system from the first work of Seiberg and Witten [2]

$$
y^{2}=\left(x^{2}-\Lambda^{4}\right)(x-u), \quad d S=\sqrt{\frac{2(u-x)}{x^{2}-\Lambda^{4}}} d x .
$$

Introduce $\lambda_{1}$ and $\lambda_{2}$ such, that $\Lambda^{2}=\lambda_{1}-\lambda_{2}$ and

$$
d S=\sqrt{\frac{2\left(\frac{\lambda_{1}+\lambda_{2}}{2}+u-x\right)}{\left(x-\lambda_{1}\right)\left(x-\lambda_{2}\right)}} d x
$$

(one can always put $\lambda_{c}=\lambda_{1}+\lambda_{2}=0$ at the end), then in follows from (2.4), that

$$
\begin{gathered}
\frac{\partial \mathcal{F}}{\partial \lambda_{1}}=\operatorname{Res}_{\lambda_{1}} \frac{2\left(\frac{\lambda_{1}+\lambda_{2}}{2}+u-x\right)}{\left(x-\lambda_{1}\right)\left(x-\lambda_{2}\right)}=\frac{\lambda_{2}-\lambda_{1}+2 u}{\lambda_{1}-\lambda_{2}} \\
\frac{\partial \mathcal{F}}{\partial \lambda_{2}}=\operatorname{Res}_{\lambda_{2}} \frac{2\left(\frac{\lambda_{1}+\lambda_{2}}{2}+u-x\right)}{\left(x-\lambda_{1}\right)\left(x-\lambda_{2}\right)}=\frac{\lambda_{1}-\lambda_{2}+2 u}{\lambda_{2}-\lambda_{1}} .
\end{gathered}
$$

Since $\frac{\partial}{\partial \lambda_{1}}=\frac{\partial}{\partial \Lambda^{2}}+\frac{\partial}{\partial \lambda_{c}}, \frac{\partial}{\partial \lambda_{2}}=-\frac{\partial}{\partial \Lambda^{2}}+\frac{\partial}{\partial \lambda_{c}}$, one gets therefore $\frac{\partial \mathcal{F}}{\partial \lambda_{c}}=1$ and

$$
\Lambda \frac{\partial \mathcal{F}}{\partial \Lambda}=4 u
$$

Substituting

$$
d \Omega=\frac{\partial d S}{\partial \Lambda^{2}}=\left(\frac{\Lambda^{2}}{x^{2}-\Lambda^{4}}+\frac{1}{4 \Lambda} \frac{\partial u}{\partial \Lambda} \frac{1}{u-x}\right) y d x
$$

into the residue formula (2.13) we get

$$
\frac{\partial^{3} \mathcal{F}}{\left(\partial \Lambda^{2}\right)^{3}}=-2 \sum_{x \in\left\{ \pm \Lambda^{2}, u\right\}} \operatorname{Res} \frac{(d \Omega)^{3}}{d x d y}=\sum_{x \in\left\{ \pm \Lambda^{2}, u\right\}} \operatorname{Res}\left(\frac{2 \Lambda^{2}}{x^{2}-\Lambda^{4}}+\frac{1}{2 \Lambda} \frac{\partial u}{\partial \Lambda} \frac{1}{u-x}\right)^{3} \frac{d x}{\left(2 / y^{2}\right)^{\prime}}
$$

and computing the residue we get an equation for the prepotential:

$$
2 \Lambda^{2}\left(\left(\frac{\partial \mathcal{F}}{\partial \Lambda}\right)^{2}-16 \Lambda^{2}\right) \frac{\partial^{3} \mathcal{F}}{\partial \Lambda^{3}}+\left(\Lambda \frac{\partial^{2} \mathcal{F}}{\partial \Lambda^{2}}-\frac{\partial \mathcal{F}}{\partial \Lambda}\right)^{3}=0
$$

It is certainly well-known (see, e.g. [32]) and even equivalent in this case to the hypergeometric differential equation for the inverse function. The only important for us thing is that it comes also from the residue formula (2.13) and allows to determine immediately the weak-coupling expansion of the prepotential, substituting an ansatz 
$\mathcal{F}=2 a^{2} \log \Lambda+\sum_{k=1}^{\infty} c_{k} \Lambda^{4 k} a^{2-4 k}$ and solving the algebraic equations for the coefficients $c_{k}$ with the result

$$
\mathcal{F}=-2 a^{2} \log a+2 a^{2} \log \Lambda+\frac{\Lambda^{4}}{4 a^{2}}+\frac{5 \Lambda^{8}}{128 a^{6}}+\frac{3 \Lambda^{12}}{128 a^{10}}+\ldots
$$

Notice only, that everywhere in this example we used the original normalization of [2] for the period $a$, which corresponds to the mass of $W$-boson.

Conformal SU(2) theory. Now, again for the illustration purposes, consider the SU(2) theory with four massless flavors, corresponding to sphere with $n=4$ marked points. Equation (2.21) acquires the form

$$
x^{2}=\frac{u}{z(z-1)(z-q)}=\frac{q(q-1) \frac{\partial \mathcal{F}}{\partial q}}{z(z-1)(z-q)}
$$

since

The residue formula

$$
\frac{\partial \mathcal{F}}{\partial q}=\operatorname{res}_{z=q} x^{2} d z=\frac{u}{q(q-1)} .
$$

$$
\left(\frac{\partial \mathcal{F}}{\partial q}\right)^{2} \frac{\partial^{3} \mathcal{F}}{\partial q^{3}}=\frac{1}{2} \operatorname{Res}_{q}\left(\left(\frac{1}{z-q}+\frac{1}{q}+\frac{1}{q-1}\right) \frac{\partial \mathcal{F}}{\partial q}+\frac{\partial^{2} \mathcal{F}}{\partial q^{2}}\right)^{3} \frac{q(q-1)(d z)^{2}}{d(z(z-1)(z-q))}
$$

gives rise to the differential equation

$$
\frac{\partial \mathcal{F}}{\partial q} \frac{\partial^{3} \mathcal{F}}{\partial q^{3}}=\frac{3}{2}\left(\frac{\partial^{2} \mathcal{F}}{\partial q^{2}}\right)^{2}+\frac{1-q+q^{2}}{2 q^{2}(q-1)^{2}}\left(\frac{\partial \mathcal{F}}{\partial q}\right)^{2}
$$

which can be rewritten in the form

$$
\{\mathcal{F}, q\}=\frac{1-q+q^{2}}{2 q^{2}(q-1)^{2}}
$$

where $\{$,$\} stays for the Schwarzian derivative. The general solution is$

$$
\mathcal{F}=\frac{A K(1-q)+B K(q)}{C K(1-q)+D K(q)}, \quad\left(\begin{array}{ll}
A & B \\
C & D
\end{array}\right) \in P S L_{2}(\mathbb{C})
$$

which comes from the fact, that $K(q)=\int_{0}^{q} \frac{d z}{\sqrt{z(z-1)(z-q)}}$ and $K(1-q)=\int_{q}^{1} \frac{d z}{\sqrt{-z(z-1)(z-q)}}$ form the basis of solutions to $f^{\prime \prime}(q)+T(q) f(q)=0$, with $T(q)=\frac{1-q+q^{2}}{2 q^{2}(q-1)^{2}}$.

To fix the physical solution we should impose, that $\mathcal{F}=a^{2} \log q+\ldots$, which gives

$$
\begin{aligned}
\mathcal{F} & =i \pi a^{2} \tau(q)=-\pi a^{2} \frac{K(1-q)}{K(q)}= \\
& =a^{2}\left(\log q-\log 16+\frac{q}{2}+\frac{13 q^{2}}{64}+\frac{23 q^{3}}{192}+\frac{2701 q^{4}}{32768}+\ldots\right) .
\end{aligned}
$$

This is just one more way to get the non-perturbative renormalization of coupling in the conformal theory with the vanishing beta-function (cf. with [22-25]). 


\subsection{Quiver gauge theory and S-duality class}

$\mathbf{S U}(2) \times \mathbf{S U}(2)$ linear quiver. Let us turn to the quiver gauge theories and consider, first, the $\mathrm{SU}(2) \times \mathrm{SU}(2)$ gauge quiver with four massless fundamentals and one bi-fundamental multiplet. We consider it in the limit $\epsilon \rightarrow 0$ after reparameterization $q_{1}=\epsilon Q_{1}, q_{2}=1-\epsilon Q_{2}$, here the parameter $\epsilon$ plays the role of degree-counting variable, so we will put $\epsilon=1$ in the final answer. The spectral curve equation (2.21) now reads

$$
\begin{aligned}
x^{2} & =\frac{q_{1}\left(q_{1}-1\right) \frac{\partial \mathcal{F}}{\partial q_{1}}}{z(z-1)\left(z-q_{1}\right)}+\frac{q_{2}\left(q_{2}-1\right) \frac{\partial \mathcal{F}}{\partial q_{2}}}{z(z-1)\left(z-q_{2}\right)}= \\
& =\frac{(1-z) F_{1}\left(1-\epsilon Q_{1}\right)+z F_{2}\left(1-\epsilon Q_{2}\right)+\epsilon^{2} Q_{1} Q_{2}\left(F_{1}+F_{2}\right)}{z\left(\epsilon Q_{1}-z\right)(z-1)\left(1-\epsilon Q_{2}-z\right)}
\end{aligned}
$$

where we parameterized the curve by $F_{1}=q_{1} \frac{\partial \mathcal{F}}{\partial q_{1}}=\frac{\partial \mathcal{F}}{\partial \log Q_{1}}$, and $F_{2}=\left(q_{2}-1\right) \frac{\partial \mathcal{F}}{\partial q_{2}}=\frac{\partial \mathcal{F}}{\partial \log Q_{2}}$.

Now we can compute the periods $a_{i}=\frac{1}{2 \pi i} \oint_{A_{i}} x d z, i=1,2$, expanding these integrals into the series. Namely,

$$
\begin{aligned}
a_{1} & =\frac{\sqrt{F_{1}}}{\pi} \int_{0}^{\epsilon Q_{1}} \sqrt{\frac{(1-z)\left(1-\epsilon Q_{1}\right)+z \frac{F_{2}}{F_{1}}\left(1-\epsilon Q_{2}\right)+\epsilon^{2} Q_{1} Q_{2}\left(1+\frac{F_{2}}{F_{1}}\right)}{(1-z)\left(1-\epsilon Q_{2}-z\right)}} \frac{d z}{\sqrt{z\left(\epsilon Q_{1}-z\right)}}= \\
& =\frac{\sqrt{F_{1}}}{\pi} \int_{0}^{\epsilon Q_{1}}\left(1+\sum_{k=1}^{\infty} f_{1, k} z^{k}\right) \frac{d z}{\sqrt{z\left(\epsilon Q_{1}-z\right)}}= \\
& =\sqrt{F_{1}}\left(1+\sum_{k=1}^{\infty} \epsilon^{k} Q_{1}^{k} \frac{(2 n-1) ! !}{(2 n) ! !} f_{1, k}\left(\epsilon Q_{1}, \epsilon Q_{2}, F_{2} / F_{1}\right)\right)
\end{aligned}
$$

where the integrals were computed using

$$
\frac{1}{\pi} \int_{0}^{\epsilon Q_{1}} \frac{z^{n} d z}{\sqrt{z\left(\epsilon Q_{1}-z\right)}}=\epsilon^{n} Q_{1}^{n} \frac{\Gamma\left(n+\frac{1}{2}\right)}{\sqrt{\pi} \Gamma(n+1)}=\epsilon^{n} Q_{1}^{n} \frac{(2 n-1) ! !}{(2 n) ! !} .
$$

The same should be done with the $A_{2}$-period

$$
\begin{aligned}
a_{2} & =\frac{\sqrt{F_{2}}}{\pi} \int_{1-\epsilon Q_{2}}^{1}\left(1+\sum_{k=1}^{\infty} f_{2, k}\left(\epsilon Q_{1}, \epsilon Q_{2}, F_{1} / F_{2}\right)(z-1)^{k}\right) \frac{d z}{\sqrt{(1-z)\left(z-1+\epsilon Q_{2}\right)}}= \\
& =\sqrt{F_{2}}\left(1+\sum_{k=1}^{\infty}(-1)^{k} \epsilon^{k} Q_{2}^{k} \frac{(2 n-1) ! !}{(2 n) ! !} f_{2, k}\left(\epsilon Q_{1}, \epsilon Q_{2}, F_{1} / F_{2}\right)\right) .
\end{aligned}
$$


Explicitly for the expansions (3.17) and (3.19), one gets

$$
\begin{aligned}
a_{1}= & \sqrt{F_{1}}-\frac{Q_{1}\left(F_{1}+F_{2}\right)}{4 \sqrt{F_{1}}} \epsilon-\frac{Q_{1}^{2}\left(7 F_{1}^{2}+14 F_{1} F_{2}+3 F_{2}^{2}\right)}{64 F_{1}^{3 / 2}} \epsilon^{2}- \\
& -\frac{Q_{1}^{2}\left(17 F_{1}^{3} Q_{1}+51 F_{1}^{2} F_{2} Q_{1}+23 F_{1} F_{2}^{2} Q_{1}+5 F_{2}^{3} Q_{1}+16 F_{1}^{2} F_{2} Q_{2}\right)}{256 F_{1}^{5 / 2}} \epsilon^{3}+\ldots \\
a_{2}= & \sqrt{F_{2}}-\frac{Q_{2}\left(F_{1}+F_{2}\right)}{4 \sqrt{F_{2}}} \epsilon-\frac{Q_{2}^{2}\left(7 F_{2}^{2}+14 F_{2} F_{1}+3 F_{1}^{2}\right)}{64 F_{2}^{3 / 2}} \epsilon^{2}- \\
& -\frac{Q_{2}^{2}\left(17 F_{2}^{3} Q_{2}+51 F_{2}^{2} F_{1} Q_{2}+23 F_{2} F_{1}^{2} Q_{2}+5 F_{1}^{3} Q_{2}+16 F_{2}^{2} F_{1} Q_{1}\right)}{256 F_{2}^{5 / 2}} \epsilon^{3}+\ldots
\end{aligned}
$$

Substituting here $F_{1}=a_{1}^{2}+\sum_{k=1}^{\infty} F_{1, k} \epsilon^{k}, F_{2}=a_{2}^{2}+\sum_{k=1}^{\infty} F_{2, k} \epsilon^{k}$ and inverting these equations, one can check, in particular, that $Q_{1} \frac{\partial F_{2}}{\partial Q_{1}}=Q_{2} \frac{\partial F_{1}}{\partial Q_{2}}$, and get the final expression for the expansion of the prepotential. It reads (after the substitution $\epsilon=1$ )

$$
\begin{aligned}
\mathcal{F}(\mathbf{a}, \mathbf{q})= & \mathcal{F}_{\text {pert }}(\mathbf{a})+a_{1}^{2} \log Q_{1}+a_{2}^{2} \log Q_{2}+\frac{a_{1}^{2}+a_{2}^{2}}{2}\left(Q_{1}+Q_{2}\right)+ \\
& +\frac{13 a_{1}^{4}+18 a_{1}^{2} a_{2}^{2}+a_{2}^{4}}{64 a_{1}^{2}} Q_{1}^{2}+\frac{a_{1}^{2}+a_{2}^{2}}{2} Q_{1} Q_{2}+\frac{13 a_{2}^{4}+18 a_{1}^{2} a_{2}^{2}+a_{1}^{4}}{64 a_{2}^{2}} Q_{2}^{2}+ \\
& +\frac{23 a_{1}^{4}+18 a_{1}^{2} a_{2}^{2}+a_{2}^{4}}{64 a_{1}^{2}} Q_{1}^{3}+\frac{13 a_{1}^{4}+18 a_{1}^{2} a_{2}^{2}+a_{2}^{4}}{64 a_{1}^{2}} Q_{1}^{2} Q_{2}+ \\
& +\frac{13 a_{2}^{4}+18 a_{1}^{2} a_{2}^{2}+a_{1}^{4}}{64 a_{2}^{2}} Q_{1} Q_{2}^{2}+\frac{23 a_{2}^{4}+18 a_{1}^{2} a_{2}^{2}+a_{1}^{4}}{64 a_{2}^{2}} Q_{2}^{3}+ \\
& +\frac{2701 a_{1}^{8}+5028 a_{1}^{6} a_{2}^{2}+470 a_{1}^{4} a_{2}^{4}-12 a_{1}^{2} a_{2}^{6}+5 a_{2}^{8}}{32768 a_{1}^{6}} Q_{1}^{4}+\frac{23 a_{1}^{4}+28 a_{1}^{2} a_{2}^{2}+3 a_{2}^{4}}{128 a_{1}^{2}} Q_{1}^{3} Q_{2}+ \\
& +\frac{17 a_{1}^{6}+343 a_{1}^{4} a_{2}^{2}+343 a_{1}^{2} a_{2}^{4}+17 a_{2}^{6}}{1024 a_{1}^{2} a_{2}^{2}} Q_{1}^{2} Q_{2}^{2}+\ldots
\end{aligned}
$$

Note that the coefficients $\frac{1}{2}, \frac{13}{64}, \frac{23}{192}, \frac{2701}{32768}$ in (3.21) are the coefficients of expansion $-\pi \frac{K(1-q)}{K(q)}$, or of the prepotential (3.15) for a single $\mathrm{SU}(2)$ group.

To fix the perturbative part one can apply the residue formula (2.13) for the a-variables

$$
\begin{aligned}
\frac{\partial^{3} \mathcal{F}_{\text {pert }}}{\partial a_{1}^{3}} & =-2 \sum_{d z=0} \operatorname{res}\left(\frac{\partial \log x}{\partial a_{1}}\right)^{3} x^{2} \frac{d z}{(\log x)^{\prime}}= \\
& =-2 \sum_{d z=0} \operatorname{res}\left(\frac{1}{2} \frac{\partial \alpha}{\partial a_{i}}-\frac{1}{2} \frac{\partial v}{\partial a_{1}} \frac{1}{z-v}\right)^{3} \frac{2 \alpha(z-v) d z}{\prod\left(z-z_{i}\right)\left(\frac{1}{z-v}-\sum \frac{1}{z-z_{i}}\right)}= \\
& =-2 \operatorname{res}_{z=v} \frac{d z}{z-v}\left(\frac{1}{2}(z-v) \frac{\partial \alpha}{\partial a_{i}}-\frac{1}{2} \frac{\partial v}{\partial a_{1}}\right)^{3} \frac{2 \alpha}{\prod\left(z-z_{i}\right)\left(1-\sum \frac{z-v}{z-z_{i}}\right)}= \\
& =\frac{1}{2}\left(\frac{\partial v}{\partial a_{1}}\right)^{3} \frac{\alpha}{\prod\left(v-z_{i}\right)}
\end{aligned}
$$


on the degenerated curve (3.16)

$$
x^{2}=\frac{(1-z) a_{1}^{2}+z a_{2}^{2}}{z\left(z-\epsilon Q_{1}\right)(z-1)\left(z-1+\epsilon Q_{2}\right)}=\frac{\alpha(z-v)}{\prod\left(z-z_{i}\right)}
$$

where we have substituted $\epsilon \rightarrow 0$ in the numerator, i.e. $F_{1}=a_{1}^{2}, F_{2}=a_{2}^{2}$. In the limit $\epsilon \rightarrow 0: v=\frac{a_{1}^{2}}{a_{1}^{2}-a_{2}^{2}}, \alpha=a_{2}^{2}-a_{1}^{2}$, i.e.

$$
\frac{\partial v}{\partial a_{1}}=-\frac{2 a_{1} a_{2}^{2}}{\left(a_{1}^{2}-a_{2}^{2}\right)^{2}}, \quad \prod\left(v-z_{i}\right)=\frac{a_{1}^{4} a_{2}^{4}}{\left(a_{1}^{2}-a_{2}^{2}\right)^{4}}
$$

and we obtain

$$
\frac{\partial^{3} \mathcal{F}_{\text {pert }}}{\partial a_{1}^{3}}=\frac{2}{a_{1}-a_{2}}+\frac{2}{a_{1}+a_{2}}-\frac{4}{a_{1}}
$$

which gives for the q-independent part

$$
\mathcal{F}_{\text {pert }}=\left(a_{1}-a_{2}\right)^{2} \log \left(a_{1}-a_{2}\right)+\left(a_{1}+a_{2}\right)^{2} \log \left(a_{1}+a_{2}\right)-2 a_{1}^{2} \log a_{1}-2 a_{2}^{2} \log a_{2} .
$$

$\mathbf{S U}(2) \times \mathbf{S U}(2) \times \mathbf{S U}(2)$ sicilian quiver. For the $\mathrm{SU}(2) \times \mathrm{SU}(2) \times \mathrm{SU}(2)$ theory the curve $(2.21)$ is parameterized as

$$
x^{2}=\frac{Q_{1}\left(\epsilon Q_{1}-1\right) \frac{\partial \mathcal{F}}{\partial Q_{1}}}{z\left(z-\epsilon Q_{1}\right)(z-1)}+\frac{\left(1-\epsilon Q_{2}\right) Q_{2} \frac{\partial \mathcal{F}}{\partial Q_{2}}}{z\left(z-1+\epsilon Q_{2}\right)(z-1)}+\frac{\left(1-\frac{1}{\epsilon Q_{3}}\right) Q_{3} \frac{\partial \mathcal{F}}{\partial Q_{3}}}{z(z-1)\left(z-\frac{1}{\epsilon Q_{3}}\right)} .
$$

We have chosen parametrization $q_{1}=\epsilon Q_{1}, q_{2}=1-\epsilon Q_{2}, q_{3}=\frac{1}{\epsilon Q_{3}}$ in the space of UV couplings to make it convenient for the computations in the weak-coupling phase for sicilian quiver with massless fundamental and (half-) tri-fundamental multiplets. This parametrization is adjusted to particular degeneration of the UV curve in such a way, that one has a single central component (corresponding to the trifundamental) connected to three another components (each corresponding to a pair of fundamentals). ${ }^{3}$

Denote again $F_{i}=Q_{i} \frac{\partial \mathcal{F}}{\partial Q_{i}}, i=1,2,3$, and solve equations for the $A$-periods of the type (3.17), (3.19). Now we need to compute one more integral $\int_{\frac{1}{\epsilon Q_{3}}}^{\infty} \frac{z^{-k-1} d z}{\sqrt{\epsilon Q_{3} z-1}}$, corresponding to the third $A$-period, which is calculated again, using formula similar to (3.18). Just the same procedure as in the case of two gauge groups leads now in a straightforward way to

\footnotetext{
${ }^{3}$ Notice, that it is essentially different from parameterization $q_{1}=\epsilon^{3} Q_{1} Q_{2} Q_{3}, q_{2}=\epsilon^{2} Q_{2} Q_{3}, q_{3}=\epsilon Q_{3}$, convenient for the computations for the linear quiver. It is easy to verify, e.g. that for these two choices of parameterization the $\epsilon \rightarrow 0$ limit is consistent with fixing homology on the curve, corresponding to particular perturbative phase of gauge theory, which in its turn is manifested by singularities in the period matrices and expansion of the perturbative prepotential.
} 
the answer

$$
\begin{aligned}
\mathcal{F}= & a_{1}^{2} \log Q_{1}+a_{2}^{2} \log Q_{2}+a_{3}^{2} \log Q_{3}-2 a_{1}^{2} \log a_{1}-2 a_{2}^{2} \log a_{2}-2 a_{3}^{2} \log a_{3}+ \\
& +\frac{1}{2} \sum_{\epsilon, \epsilon^{\prime}= \pm}\left(a_{1}+\epsilon a_{2}+\epsilon^{\prime} a_{3}\right)^{2} \log \left(a_{1}+\epsilon a_{2}+\epsilon^{\prime} a_{3}\right)+ \\
& +\frac{a_{1}^{2}+a_{2}^{2}-a_{3}^{2}}{2} Q_{1}+\frac{a_{1}^{2}+a_{2}^{2}-a_{3}^{2}}{2} Q_{2}+\frac{-a_{1}^{2}+a_{2}^{2}+a_{3}^{2}}{2} Q_{3}+ \\
& +\frac{a_{1}^{2}+a_{2}^{2}-a_{3}^{2}}{4} Q_{1} Q_{2}+\frac{a_{1}^{2}-a_{2}^{2}-a_{3}^{2}}{4} Q_{2} Q_{3}+\frac{a_{1}^{2}-a_{2}^{2}+a_{3}^{2}}{4} Q_{1} Q_{3}+ \\
& +\frac{13 a_{1}^{4}+18 a_{1}^{2} a_{2}^{2}-14 a_{1}^{2} a_{3}^{2}+a_{2}^{4}-2 a_{2}^{2} a_{3}^{2}+a_{3}^{4}}{64 a_{1}^{2}} Q_{1}^{2}+ \\
& +\frac{a_{1}^{4}+13 a_{2}^{4}-14 a_{2}^{2} a_{3}^{2}+a_{3}^{4}+18 a_{1}^{2} a_{2}^{2}-2 a_{1}^{2} a_{3}^{2}}{64 a_{2}^{2}} Q_{2}^{2}+ \\
& +\frac{a_{1}^{4}+a_{2}^{4}+18 a_{2}^{2} a_{3}^{2}+13 a_{3}^{4}-2 a_{1}^{2} a_{2}^{2}-14 a_{1}^{2} a_{3}^{2}}{64 a_{3}^{2}} Q_{3}^{2}+\ldots
\end{aligned}
$$

Let us stress here, that the result in this case, where the standard methods [15] of the instanton calculus are not applicable directly [16], is obtained from the study of gaugetheory tau-functions exactly in the same way as for the theories, where the weak-coupling expansion is saturated by the instanton configurations. This allows us to hope for a direct application of our methods for the S-duality class of generic $\mathrm{SU}(N)$ quiver gauge theories, which can shed light to the physical properties of supersymmetric gauge theories, which do not even have a Lagrangian formulation.

\subsection{Mass-deformed theory and quasiclassical conformal block}

For the $n=4$ massless $\mathrm{SU}(2)$ theory the prepotential is given by quadratic expression (3.15). Consider now its simplest deformation, when two flavors receives an opposite masses, e.g. $\Delta_{0}=m^{2}, \Delta_{1}=\Delta_{q}=\Delta_{\infty}=0$, with the curve (2.21) for this case

$$
x^{2}=\frac{z q(q-1) \mathcal{F}^{\prime}-(z-q) m^{2}}{z^{2}(z-1)(z-q)}
$$

where $\mathcal{F}^{\prime}=\frac{\partial \mathcal{F}}{\partial q}$. The residue formula (2.14) gives now the differential equation

$$
\begin{aligned}
\mathcal{F}^{\prime \prime \prime} & +\frac{m^{2} \mathcal{F}^{\prime}\left[3 q(2-3 q) \mathcal{F}^{\prime \prime}+2(1-3 q) \mathcal{F}^{\prime}\right]-\left[3 q^{4}(q-1)^{2} \mathcal{F}^{\prime 2} \mathcal{F}^{\prime \prime 2}+q^{2}\left(q^{2}-q+1\right) \mathcal{F}^{\prime 4}\right]}{2 q^{2}(q-1) \mathcal{F}^{\prime}\left[q^{2}(q-1) \mathcal{F}^{\prime 2}+m^{2} q(q-2) \mathcal{F}^{\prime}-m^{4}\right]}- \\
& -\frac{m^{4}\left[(q-1)^{2} q^{2} \mathcal{F}^{\prime \prime 3}+6 q(q-1)^{2} \mathcal{F}^{\prime} \mathcal{F}^{\prime \prime 2}+3\left(q^{2}+q-1\right) \mathcal{F}^{\prime \prime} \mathcal{F}^{\prime 2}+(3+2 q) \mathcal{F}^{\prime 3}\right]}{2(q-1) \mathcal{F}^{\prime}\left[q^{2}(q-1) \mathcal{F}^{\prime 2}+m^{2} q(q-2) \mathcal{F}^{\prime}-m^{4}\right]}=0
\end{aligned}
$$

which can be solved, using the anzatz $\mathcal{F}=\left(a^{2}-m^{2}\right) \log q+\sum_{k=1}^{\infty} c_{k} q^{k}$, giving rise to expansion

$$
\begin{aligned}
\mathcal{F}= & \mathcal{F}_{\text {pert }}(a ; m)+\left(a^{2}-m^{2}\right) \log q+\frac{a^{2}-m^{2}}{2} q+ \\
& +\frac{13 a^{4}-14 a^{2} m^{2}+m^{4}}{64 a^{2}} q^{2}+\frac{23 a^{4}-26 a^{2} m^{2}+3 m^{4}}{192 a^{2}} q^{3}+ \\
& +\frac{2701 a^{8}-3164 a^{6} m^{2}+470 a^{4} m^{4}-12 a^{2} m^{2}+5 m^{8}}{32768 a^{6}} q^{4}+\ldots
\end{aligned}
$$


Notice, that this prepotential is directly related to the corresponding expression (3.21) in the massless $\mathrm{SU}(2) \times \mathrm{SU}(2)$ theory (we compare $\mathcal{F}(a, m, q)$ for a single massive flavor theory with the massless prepotential $\mathcal{F}\left(a_{1}, a_{2}, q_{1}, 1-Q_{2}\right)$ for two gauge groups (3.21) in the limit $\left.Q_{2}=0, a_{2}=m\right)$. Their difference

$$
\left[\left.\mathcal{F}(a, \tilde{a}, q, 1-Q)\right|_{\tilde{a}=m}-m^{2} \log Q\right]_{Q=0}-\mathcal{F}(a, m, q)=-m^{2} \log (1-q)
$$

is just a $\mathrm{U}(1)$-factor, commonly arising in the context of the AGT correspondence [7]. The q-independent term in (3.31)

$$
\mathcal{F}_{\text {pert }}(a ; m)=(a-m)^{2} \log (a-m)+(a+m)^{2} \log (a+m)-2 a^{2} \log a
$$

is restored in standard way from residue formula on degenerate curve, and it vanishes in the limit $m \rightarrow 0$.

Now let us add more massive deformations for a single $\mathrm{SU}(2)$ and consider generic four-point function

$$
\begin{aligned}
x^{2} & =\frac{(q-1) q \mathcal{F}^{\prime}}{z(z-q)(z-1)}+\frac{\Delta_{0}}{z^{2}}+\frac{\Delta_{1}}{(z-1)^{2}}+\frac{\Delta_{q}}{(z-q)^{2}}-\frac{\Delta_{0}+\Delta_{1}+\Delta_{q}-\Delta_{\infty}}{z(z-1)}= \\
& =\frac{Q_{4}(z)}{z^{2}(z-1)^{2}(z-q)^{2}}=\phi_{2}(z)
\end{aligned}
$$

where four $\Delta=\Delta(\mathbf{m})$ are quadratic functions of the fundamental masses only. Denote $\left.q \mathcal{F}^{\prime}\right|_{q=0}=A$ and look first for the solution in the weak-coupling region $q \rightarrow 0$. One has

$$
\begin{aligned}
Q_{4}^{(0)}(z) & =\left.Q_{4}(z)\right|_{q=0}=z^{2}\left(\Delta_{\infty} z^{2}+\left(-A-\Delta_{0}+\Delta_{1}-\Delta_{q}-\Delta_{\infty}\right) z+\left(A+\Delta_{0}+\Delta_{q}\right)\right) \\
\frac{\partial x}{\partial a} & =\frac{1}{2 \sqrt{Q_{4}(z)}} \frac{\partial Q(z)}{\partial a} \frac{1}{z(z-1)(z-q)} \underset{q \rightarrow 0}{\approx}-\frac{\frac{\partial A}{\partial a}}{2 \sqrt{Q_{4}^{(0)}(z)}}
\end{aligned}
$$

and from the normalization of the holomorphic differential

$$
1 \approx-\frac{1}{4 \pi i} \frac{\partial A}{\partial a} \oint_{A} \frac{d z}{\sqrt{Q_{4}^{(0)}(z)}} \approx-\frac{1}{2} \frac{\partial A}{\partial a} \frac{1}{\sqrt{A+\Delta_{0}+\Delta_{q}}}
$$

which gives $A=a^{2}-\Delta_{0}-\Delta_{q}$, i.e. the leading exponent for $q \rightarrow 0$ expansion of the four-point conformal block on sphere.

The differential equation is obtained similarly to (3.30), though it requires for generic massive deformation some additional efforts — to sum in the residue formula

$$
\mathcal{F}^{\prime \prime \prime}=-\operatorname{res}_{z=q, Q_{4}(z)=0} \frac{\left(\phi_{2}^{\prime}\right)^{3} d z}{2 \phi_{2} \frac{d \phi_{2}}{d z}}=-\operatorname{res}_{z=q, Q_{4}(z)=0} \frac{T(z) d z}{Q_{4}(z) S(z)}
$$

over the unknown roots of the polynomial $Q_{4}(z)$ in the equation (3.34) in addition to the fourth-order pole at $S(z) \underset{z \rightarrow q}{\sim}(z-q)^{4}$. Calculating the sum over the zeroes of a polynomial $Q_{k}(z)=\prod_{i=1}^{k}\left(z-\lambda_{i}\right)$

$$
\sum_{i=1}^{k} \frac{T\left(\lambda_{i}\right)}{S\left(\lambda_{i}\right) Q^{\prime}\left(\lambda_{i}\right)}=\frac{\sum_{i=1}^{k} T\left(\lambda_{i}\right) \prod_{j \neq i}\left(Q^{\prime}\left(\lambda_{j}\right) S\left(\lambda_{j}\right)\right)}{\prod_{i=1}^{k} S\left(\lambda_{i}\right) Q^{\prime}\left(\lambda_{i}\right)}=\frac{\sum_{i=1}^{k} T\left(\lambda_{i}\right) \prod_{j \neq i}\left(Q^{\prime}\left(\lambda_{j}\right) S\left(\lambda_{j}\right)\right)}{\mathrm{R}\{S, Q\} \mathrm{D}\{Q\}}
$$


where $\mathrm{R}\{S, Q\}$ is the resultant and $\mathrm{D}\{Q\}$ stays for the discriminant, one gets some rational symmetric function of the roots of $Q_{4}(z)$.

Once the differential equation was derived, we substitute the perturbative expansion $\mathcal{F}=A \log q+\sum_{i=1}^{\infty} c_{i} q^{i}$ and obtain an answer for the prepotential (here the result for $\Delta_{0}=\Delta_{q}=0$ and arbitrary $\Delta_{1}=m_{1}^{2}$ and $\Delta_{\infty}=m_{\infty}^{2}$ is presented $\left.{ }^{4}\right)$ :

$$
\begin{aligned}
\mathcal{F}= & a^{2} \log q+\frac{1}{2} \sum_{\epsilon, \epsilon^{\prime}}\left(a+\epsilon m_{1}+\epsilon^{\prime} m_{\infty}\right)^{2} \log \left(a+\epsilon m_{1}+\epsilon^{\prime} m_{\infty}\right)-2 a^{2} \log a+ \\
& +\frac{a^{2}+m_{1}^{2}-m_{\infty}^{2}}{2} q+\frac{13 a^{4}+18 a^{2} m_{1}^{2}-14 a^{2} m_{\infty}^{2}+m_{1}^{4}+m_{\infty}^{4}-2 m_{1}^{2} m_{\infty}^{2}}{64 a^{2}} q^{2}+ \\
& +\frac{207 a^{6}+a^{4}\left(334 m_{1}^{2}-226 m_{\infty}^{2}\right)+a^{2}\left(43 m_{1}^{4}-54 m_{1}^{2} m_{\infty}^{2}+11 m_{\infty}^{4}\right)-8\left(m_{1}^{2}-m_{\infty}^{2}\right)^{3}}{1728 a^{4}} q^{3}+ \\
& +O\left(q^{4}\right) .
\end{aligned}
$$

It is instructive to compare this result with the expansion for the quasiclassical conformal block from [33], depending on intermediate dimension $\Delta=a^{2}$ in addition to the external dimensions. For two non-vanishing external dimensions, as in (3.40), the formula for quasiclassical conformal block gives

$$
\begin{aligned}
f(q)= & a^{2} \log q+\frac{a^{2}+m_{1}^{2}-m_{\infty}^{2}}{2} q+\left(\frac{a^{2}+m_{1}^{2}-m_{\infty}^{2}}{4}+\right. \\
& \left.+\frac{a^{4}+2 a^{2}\left(m_{1}^{2}+m_{\infty}^{2}\right)-3\left(m_{1}^{2}-m_{\infty}^{2}\right)^{2}}{64\left(a^{2}+\frac{3}{4}\right)}-\frac{a^{4}-\left(m_{1}^{2}-m_{\infty}^{2}\right)^{2}}{16 a^{2}}\right) q^{2}+\ldots
\end{aligned}
$$

and the single mass case is easily reproduced by $m_{\infty} \mapsto m \neq 0, m_{1} \mapsto 0$. It is easy to see, that expressions (3.40) and (3.41) literally coincide in the SW limit for conformal blocks, when all dimensions $\Delta \rightarrow \infty$, including intermediate, simultaneously. Then almost all terms remain intact except for $\frac{3}{4 \Delta} \rightarrow 0$, and the correction in denominator from the inverse Shapovalov form disappear.

In convenient parametrization for two-dimensional conformal theory $\epsilon_{1}=b g, \epsilon_{2}=\frac{g}{b}$, the central charge is $c=1+6 \frac{\left(\epsilon_{1}+\epsilon_{2}\right)^{2}}{\epsilon_{1} \epsilon_{2}}=1+6\left(b+\frac{1}{b}\right)^{2}$, and for conformal dimensions one can write $\Delta(\alpha)=\frac{\left(\epsilon_{1}+\epsilon_{2}\right)^{2}}{4 \epsilon_{1} \epsilon_{2}}-\frac{\alpha^{2}}{\epsilon_{1} \epsilon_{2}}=\frac{1}{4}\left(b+\frac{1}{b}\right)^{2}-\frac{\alpha^{2}}{g^{2}}$. The quasiclassical limit corresponds to $b=g \rightarrow 0$, which means $\epsilon_{2}=1, \epsilon_{1} \rightarrow 0$. The SW limit corresponds to $c \ll \Delta$, so we should put $\frac{b}{g} \gg 1$ and $g \ll 1$, therefore in this limit both $\epsilon_{1} \rightarrow 0, \epsilon_{2} \rightarrow 0$. In this limit our prepotential receives the $\mathrm{U}(1)_{\mathcal{R}}$ symmetry, which was broken by some integer numbers in two-dimensional conformal theory. It is still a nontrivial question about the limit of Painleve VI in such case. We hope to return to this issue elsewhere.

\footnotetext{
${ }^{4}$ From the physical point of view, as in $(3.33)$, the perturbative part is a result of partial cancelation

$$
\mathcal{F}_{\text {pert }}=\frac{1}{2} \sum_{\epsilon, \epsilon^{\prime}}\left[\left(a+\epsilon m_{0}+\epsilon^{\prime} m_{q}\right)^{2} \log \left(a+\epsilon m_{0}+\epsilon^{\prime} m_{q}\right)+\left(a+\epsilon m_{1}+\epsilon^{\prime} m_{\infty}\right)^{2} \log \left(a+\epsilon m_{1}+\epsilon^{\prime} m_{\infty}\right)\right]-
$$$$
-4 a^{2} \log a
$$

between the contribution of massless fundamental and vector multiplets at $m_{0}=m_{q}=0$. 


\section{Zamolodchikov's conformal blocks}

The AGT conjecture [7] allows to apply the techniques of four-dimensional supersymmetric gauge theories to answer to some complicated questions of two-dimensional conformal theory (see, e.g. [44]) and vice versa. In the SW limit $\epsilon_{1}, \epsilon_{2} \rightarrow 0$ one can identify the extended prepotentials to certain limit of the $c=1$ conformal blocks, and if the conformal dimensions are fixed on the two-dimensional side, it just corresponds to vanishing of the masses of external multiplets. The SW formulation, if $\Sigma_{0}$ is a sphere with punctures, leads to the set of differential equations for the conformal blocks in such limit, while the underlying geometry is the $g$-parametric family of genus $g$ curves.

It is interesting to compare this description for the $\mathrm{SU}(2)$-quiver gauge theories with another well-known case, proposed by Al. Zamolodchikov for the conformal blocks of $c=1$ Ashkin-Teller model [18], and described in very similar terms. The Zamolodchikov result for a $2 g+2$-point conformal block for the spin fields with external dimensions $\frac{1}{16}$ was given in terms of a a single genus- $g$ curve, and required an extra charge-conservation constrains for the dimensions in the internal vertices of the block. Solving equation $2 g+2=g+3$, one gets $g=1$, corresponding to the four-point conformal block and conformal SU(2) supersymmetric QCD (3.9), (3.15), where these two constructions obviously coincide. In general situation, there is a difference, whose origin comes from the vanishing of some (half-) trifundamental masses - in the triple-vertices. For the Zamolodchikov conformal blocks this is just charge conservation in $c=1$ conformal theory, which is certainly absent for generic $\mathrm{SU}(2)$ quiver theory on the gauge theory side. Hence, in the SW approach it is equivalent to the extra relations on the condensates for three gauge groups, interacting with the same tri-fundamental multiplet of matter. The first time, when such conservation law can be imposed is the case of sicilian quiver with the curve (3.27) and the tau-function (3.28), the Zamolodchikov constraint is singular from the point of view of four-dimensional physics (vanishing of one of the multiplet masses, which has been already integrated out to get the SW effective action), but the prepotential (3.28) is regular in this limit, and becomes just a quadratic function of the condensates, in accordance with [18].

Another reason to discuss this case, which is explicitly solvable even on the CFT side of the correspondence, is that there exists also the isomonodromic-CFT correspondence [3742 , with an exact solution for the $2 g+2$-point isomonodromic tau-function of the special type [43], related to the Zamolodchikov conformal block. So the constrained case of the sicilian quiver and other gauge theories with massless fundamental and partially massless tri-fundamental matter turns to be exactly-solvable in three different approaches. Note also, that this case on gauge-theory side is the simplest example of the S-duality class, where the standard methods of instanton calculus are not applicable [16], so the correspondence between the four-dimensional and two-dimensional sides goes in fact even beyond the standard formulation [7] of the AGT-correspondence.

A generic Zamolodchikov case corresponds to a special case of the $n=g+3$ point conformal block with $V=|\mathcal{V}|=\frac{1}{2} n-2$ triple vertices $\left\{\mathcal{V}_{i} \in \mathcal{V}\right\}$ or half-tri-fundamental multiplets ( $n$ must be even in this case). At each such vertex $c=1$ conformal theory gives 
one conservation condition, so that the genus drops to

$$
\tilde{g}=g-V=n-3-\left(\frac{1}{2} n-2\right)=\frac{1}{2} n-1
$$

and for the total number of punctures we restore $n=2 \tilde{g}+2 .{ }^{5}$ Another form

$$
\tilde{g}-1=\frac{g-1}{2}
$$

of the same relation (4.1) means that the Euler characteristic $\chi(\tilde{\Sigma})=\frac{1}{2} \chi(\Sigma)$ decreases twice after the degeneration.

We are going now to present the direct proof, that in such limit the extended SW prepotential (2.1), (2.4) becomes the quadratic form

$$
\left.\mathcal{F}(\mathbf{a}, \mathbf{q})\right|_{\bigcup_{\mathcal{V}} \sum_{i \in \mathcal{V}_{i}} a_{i}=0}=i \pi \sum_{\alpha, \beta=1}^{\tilde{g}} a_{\alpha} \mathcal{T}_{\alpha \beta}(\mathbf{q}) a_{\beta}
$$

with the period matrix $\mathcal{T}=\left\|\mathcal{T}_{\alpha \beta}\right\|$ of the hyperelliptic curve $\tilde{\Sigma}$ of genus $\tilde{g}$, which does not depend on the condensates (the moduli space of this hyperelliptic curve is parameterized by original set of the UV couplings). This result has been obtained originally, using the language of free field on Riemann surface.

Consider now the massless $\mathrm{SU}(2)$ quiver theory with the generating differential

$$
\begin{aligned}
d S=x d z & =\sqrt{\alpha} \frac{\sqrt{\prod_{k=1}^{g-1}\left(z-v_{k}\right)} d z}{\sqrt{\prod_{j=1}^{g+3}\left(z-z_{j}\right)}} \quad\left(z_{\left.1, \ldots, z_{g+3}\right) \rightarrow\left(q_{1}, \ldots, q_{g}, 1, \infty, 0\right)}^{\longrightarrow}\right. \\
& \rightarrow \sqrt{\alpha} \frac{\sqrt{\prod_{k=1}^{g-1}\left(z-v_{k}\right)} d z}{\sqrt{z(z-1) \prod_{j=1}^{g}\left(z-q_{j}\right)}}
\end{aligned}
$$

on a hyperelliptic curve (2.21) of genus $g$, with the total number of branch points (from both numerator and denominator) \#B.P. $=2 g+2$. Impose now

$$
\frac{g-1}{2}=\frac{1}{2} n-2=V
$$

constraints to the coefficients $\left\{v_{j}\right\}, j=1, \ldots, g-1$ in the numerator of (4.4) in order to get the total square, i.e.

$$
d S=x d z \rightarrow \frac{\mathcal{Q}_{\tilde{g}-1}(z) d z}{y}
$$

\footnotetext{
${ }^{5}$ From now on we will denote by $\tilde{g}$ the genus of hyperelliptic curve in the constrained case. Due to the conservation conditions the number of remaining independent gauge theory condensates $\tilde{g}$ will be always less in Zamolodchikov's case than the amount $n-3=2 \tilde{g}-1$ of the UV couplings.
} 
with some polynomial $\mathcal{Q}_{\tilde{g}-1}(z)$ of power $\tilde{g}-1$, which can be considered as a holomorphic differential on the "reduced" hyperelliptic curve $\tilde{\Sigma}$ :

$$
\begin{aligned}
y^{2} & =\prod_{j=1}^{g+3}\left(z-z_{j}\right)_{\left(z_{1}, \ldots, z_{g+3}\right) \rightarrow\left(q_{1}, \ldots, q_{g}, 1, \infty, 0\right)}^{\longrightarrow} \\
\rightarrow y^{2} & =z(z-1) \prod_{k=1}^{g}\left(z-q_{k}\right)=z(z-1) \prod_{k=1}^{2 \tilde{g}-1}\left(z-q_{k}\right)
\end{aligned}
$$

already of genus (4.1). The differential (4.6) can be decomposed

$$
d S=\frac{\mathcal{Q}_{\tilde{g}-1}(z) d z}{y}=\sum_{\alpha=1}^{\tilde{g}} a_{\alpha} \frac{R_{\alpha}(z) d z}{y}=\sum_{\alpha=1}^{\tilde{g}} a_{\alpha} d \omega_{\alpha}
$$

into a linear combination of the normalized holomorphic differentials on (4.7), so that the system of linear equations

$$
\frac{1}{2 \pi i} \oint_{A_{\alpha}} \frac{\mathcal{Q}_{\tilde{g}-1}(z) d z}{y}=a_{\alpha}, \quad \alpha=1, \ldots, \tilde{g}
$$

can be solved for $\tilde{g}$ coefficients of the polynomial $\mathcal{Q}_{\tilde{g}-1}(z)$. Equivalently, the system of equations

$$
\frac{1}{2 \pi i} \oint_{A_{\alpha}} \frac{R_{\beta}(z) d z}{y}=\delta_{\alpha \beta}, \quad \alpha, \beta=1, \ldots, \tilde{g}
$$

fixes all $\tilde{g}^{2}$ coefficients of the polynomials $\left\{R_{\alpha}(z)\right\}$ of power $\tilde{g}-1$, defining the normalized holomorphic differentials

$$
d \omega_{\alpha}=\frac{R_{\alpha}(z) d z}{y}, \quad \alpha=1, \ldots, \tilde{g}
$$

in (4.8). The solution to the dual period equations

$$
\frac{\partial \mathcal{F}}{\partial a_{\alpha}}=\oint_{B_{\alpha}} \frac{\mathcal{Q}_{\tilde{g}-1}(z) d z}{y}=2 \pi i \sum_{\beta=1}^{\tilde{g}} a_{\beta} \oint_{B_{\alpha}} d \omega_{\beta}=2 \pi i \sum_{j=1}^{\tilde{g}} \mathcal{T}_{\alpha \beta}(\mathbf{q}) a_{\beta}
$$

immediately gives rise to the formula (4.3) with the period matrix of (4.7), up to an a-independent constant. Relations to the dependence of the reduced prepotential upon the ramification points (2.4), i.e.

$$
\begin{aligned}
\frac{\partial \mathcal{F}}{\partial q_{i}} & =\operatorname{res}_{q_{i}} \frac{(d S)^{2}}{d z}=\frac{\mathcal{Q}_{\tilde{g}-1}^{2}\left(q_{i}\right)}{q_{i}\left(q_{i}-1\right) \prod_{j \neq i}\left(q_{i}-q_{j}\right)}= \\
& =\sum_{\alpha, \beta=1}^{\tilde{g}} a_{\alpha} a_{\beta} \frac{R_{\alpha}\left(q_{i}\right) R_{\beta}\left(q_{i}\right)}{q_{i}\left(q_{i}-1\right) \prod_{j \neq i}\left(q_{i}-q_{j}\right)}, \quad i=1, \ldots, 2 \tilde{g}-1
\end{aligned}
$$

immediately comes from (4.8) and completely fixed (4.3) up to a constant. This exactly coincides with the Zamolodchikov equation [18] for the leading contribution to the correlator $e^{\mathcal{F}}=\left\langle\sigma_{0}\left(z_{1}\right) \ldots \sigma_{0}\left(z_{n}\right)\right\rangle$ of spin fields in the AT model, see appendix A. Below we shall 
also use it to prove the nonlinear relations, arising from the SW theory to the derivatives of the matrix elements of the period matrix of hyperelliptic curves.

Results of this section are in complete agreement with the above analysis of the weakcoupling expansions for the quiver tau-functions. Already from the perturbative part of (3.28) we see, that in Zamolodchikov's limit for $\mathrm{SU}(2) \times \mathrm{SU}(2) \times \mathrm{SU}(2)$ prepotential the expression for period matrix of $\Sigma$ becomes singular, when $a_{1} \pm a_{2} \pm a_{3}=0$ (vanishing mass of one of the states from the (half-) tri-fundamental multiplet). It means that the curve $\Sigma$ indeed degenerates to $\tilde{\Sigma}$, and it is easy to see, that all denominators in (3.28) disappear in this limit and the prepotential turns into a quadratic expression in the remaining SW periods.

The Zamolodchikov case extends the example of the SU(2) conformal theory with elliptic curve (3.9) to a subfamily of quiver gauge theories which are non-renormalized within the perturbation theory, i.e. have vanishing beta-functions, ${ }^{6}$ but the true IR couplings are renormalized by the non-perturbative effects. We are going to show in next section, that equations (4.13) are immediately rewritten in the form of differential equations for effective couplings (the derivatives are taken over the bare couplings, since there are no other parameters in the theory), which take the form of the Rauch relations, and can be implicitly solved via the Thomae formulas $[45,46]$ (see also [47, 48]).

\section{Non-linear equations in quiver gauge theory}

In section 3 we have already used the differential equations, coming from the relations on quasiclassical tau-functions $[8,9]$, to get the weak-coupling expansions for the supersymmetric gauge theories. Particular examples of such equations (see e.g. (3.13)), and the direct relation of these equations to quasiclassical expansions of the conformal blocks (and therefore to Painleve VI) show that they have indeed some deep geometric origin. Below in this section, we are going to study the differential equations, arising from the SW approach to quiver gauge theories, in more general context.

In the constrained Zamolodchikov's case all equations for the prepotential can be rewritten as relations to the period matrices of hyperelliptic curves. We are going to show, that all such relations for the first derivatives are actually consequences of the Rauch formulas. They propose some parametrization in the space of first derivatives, which can be studied in algebro-geometric terms.

Another natural thing is to expect the WDVV-like equations [29] to be satisfied by extended prepotentials of the quiver gauge theories. We prove indeed, that such equations are satisfied by quiver tau-functions in the massless case as functions of the whole set of variables: all SW periods and bare couplings. Amazingly enough, the proof, based on the residue formula (2.13) and a simple counting argument $[35,36]$, is valid both in the unconstrained and Zamolodchikov's cases, leading in the latter situation to the relations including the third derivatives of the period matrices.

\footnotetext{
${ }^{6}$ More strictly, the perturbative calculations give rise only to a finite renormalization of the couplings.
} 


\subsection{Relations for the period matrix}

Consider, first, the simplest example of our SW system - the Zamolodchikov case of section 4, represented by hyperelliptic curve (4.7), parameterized by the couplings q only, with the holomorphic SW differential (4.8). The SW equations (2.1) are trivially solved, but the formula (2.4) is still non-trivial (see (4.12), (4.3) and (4.13)).

Comparing the coefficients of the quadratic forms at the both sides of (4.13), one gets for the first derivatives of the period matrix of hyperelliptic curve (4.7)

$$
\frac{\partial \mathcal{T}_{\alpha \beta}(\mathbf{q})}{\partial q_{k}}=\operatorname{res}_{q_{k}} \frac{d \omega_{\alpha} d \omega_{\beta}}{d z}=\frac{R_{\alpha}\left(q_{k}\right) R_{\beta}\left(q_{k}\right)}{q_{k}\left(q_{k}-1\right) \prod_{l \neq k}\left(q_{k}-q_{l}\right)}, \quad k=1, \ldots, 2 \tilde{g}-1
$$

in terms of the numerators for the holomorphic differentials in (4.11): it is exactly one of the well-known Rauch formulas [45]. Their solution can be found via the Thomae formulas $[45,46]$, which can be written for the curve (4.7) in the form

$$
q_{k}^{2}= \pm \frac{\theta\left[\eta_{1}\right](\mathcal{T})^{4} \theta\left[\eta_{2}\right](\mathcal{T})^{4}}{\theta\left[\eta_{3}\right](\mathcal{T})^{4} \theta\left[\eta_{4}\right](\mathcal{T})^{4}}, \quad k=1, \ldots, 2 \tilde{g}-1
$$

for the set of four theta-characteristics, chosen in the following way. Divide the branch points as

$$
\left\{z_{1}, \ldots, z_{n}\right\}=\{0,1, \infty, k\} \sqcup S^{\prime} \sqcup S^{\prime \prime}
$$

where $S^{\prime} \supset\left\{q_{j^{\prime}}\right\}$ and $S^{\prime \prime} \supset\left\{q_{j^{\prime \prime}}\right\}$ are any two nonintersecting sets, containing each $\tilde{g}-1$ branch points with $j^{\prime} \neq k, j^{\prime \prime} \neq k$ and $j^{\prime} \neq j^{\prime \prime}$. Then

$$
\begin{aligned}
& \eta_{1}=\left\{S^{\prime} \oplus k \oplus \infty\right\} \sqcup\left\{S^{\prime \prime} \oplus 0 \oplus 1\right\} \\
& \eta_{2}=\left\{S^{\prime} \oplus 0 \oplus 1\right\} \sqcup\left\{S^{\prime \prime} \oplus k \oplus \infty\right\} \\
& \eta_{3}=\left\{S^{\prime} \oplus 0 \oplus k\right\} \sqcup\left\{S^{\prime \prime} \oplus 1 \oplus \infty\right\} \\
& \eta_{4}=\left\{S^{\prime} \oplus 1 \oplus \infty\right\} \sqcup\left\{S^{\prime \prime} \oplus 0 \oplus k\right\}
\end{aligned}
$$

are possible choices of even theta-characteristics in (5.2) in terms of partitions of the branch points. The proof of this fact can be found, for example, in [45, 47, 48].

As an example, consider the first nontrivial Zamolodchikov's case with $\tilde{g}=2$, i.e.

$$
x^{2}=\sum_{i=1}^{3} \frac{q_{i}\left(q_{i}-1\right)}{z(z-1)\left(z-q_{i}\right)} \frac{\partial \mathcal{F}}{\partial q_{i}}=\frac{\alpha\left(z-z_{0}\right)^{2}}{z(z-1)\left(z-q_{1}\right)\left(z-q_{2}\right)\left(z-q_{3}\right)} .
$$

Expression in the r.h.s. means, that there should one relation for the q-derivatives of prepotential. It can be obtained by calculating the discriminant and leads to the algebraic equation

$$
\xi_{1}^{2}+\xi_{2}^{2}+\xi_{3}^{2}-2 \xi_{1} \xi_{2}-2 \xi_{2} \xi_{3}-2 \xi_{1} \xi_{3}=0
$$

for the variables

$$
\begin{aligned}
& \xi_{1}=\left(q_{2}-q_{3}\right) q_{1}\left(q_{1}-1\right) \frac{\partial \mathcal{F}}{\partial q_{1}}, \quad \xi_{2}=\left(q_{3}-q_{1}\right) q_{2}\left(q_{2}-1\right) \frac{\partial \mathcal{F}}{\partial q_{2}} \\
& \xi_{3}=\left(q_{1}-q_{2}\right) q_{3}\left(q_{3}-1\right) \frac{\partial \mathcal{F}}{\partial q_{3}}
\end{aligned}
$$


and becomes an identity after using the Rauch formulas, or just substituting

$$
\begin{aligned}
\xi_{1} & =\frac{q_{2}-q_{3}}{\left(q_{1}-q_{2}\right)\left(q_{2}-q_{3}\right)} \mathcal{Q}\left(q_{1}\right)^{2}, \quad \xi_{2}=\frac{q_{3}-q_{1}}{\left(q_{2}-q_{1}\right)\left(q_{2}-q_{3}\right)} \mathcal{Q}\left(q_{2}\right)^{2} \\
\xi_{3} & =\frac{q_{1}-q_{2}}{\left(q_{3}-q_{1}\right)\left(q_{3}-q_{2}\right)} \mathcal{Q}\left(q_{3}\right)^{2}
\end{aligned}
$$

for any linear $\mathcal{Q}_{\tilde{g}-1}=\mathcal{Q}(z)$. Equation (5.5) also expresses

$$
\alpha=\frac{\partial \mathcal{F}}{\partial q_{1}} q_{1}\left(q_{1}-1\right)+\frac{\partial \mathcal{F}}{\partial q_{2}} q_{2}\left(q_{2}-1\right)+\frac{\partial \mathcal{F}}{\partial q_{3}} q_{3}\left(q_{3}-1\right)=K_{\alpha}\left(a_{1}, a_{2}\right)
$$

and

$$
\begin{aligned}
z_{0} & =-\frac{1}{2} \frac{\frac{\partial \mathcal{F}}{\partial q_{1}} q_{1}\left(q_{1}-1\right)\left(q_{2}+q_{3}\right)+\frac{\partial \mathcal{F}}{\partial q_{2}} q_{2}\left(q_{2}-1\right)\left(q_{1}+q_{3}\right)+\frac{\partial \mathcal{F}}{\partial q_{3}} q_{3}\left(q_{3}-1\right)\left(q_{1}+q_{2}\right)}{\frac{\partial \mathcal{F}}{\partial q_{1}} q_{1}\left(q_{1}-1\right)+\frac{\partial \mathcal{F}}{\partial q_{2}} q_{2}\left(q_{2}-1\right)+\frac{\partial \mathcal{F}}{\partial q_{3}} q_{3}\left(q_{3}-1\right)}= \\
& =-\frac{1}{2} \frac{K_{z}\left(a_{1}, a_{2}\right)}{K_{\alpha}\left(a_{1}, a_{2}\right)}
\end{aligned}
$$

in terms of quadratic forms in the SW periods with the coefficients

$$
\begin{aligned}
K_{\alpha}^{i j} & =\sum_{k=1}^{3} q_{k}\left(q_{k}-1\right) \frac{\partial \mathcal{T}_{i j}}{\partial q_{k}}=\sum_{k=1}^{3} q_{k}\left(q_{k}-1\right) \frac{\partial^{3} \mathcal{F}}{\partial q_{k} \partial a_{i} \partial a_{j}} \\
K_{z}^{i j} & =\left(q_{1}+q_{2}+q_{3}\right) K_{\alpha}^{i j}-\sum_{k=1}^{3} q_{k}^{2}\left(q_{k}-1\right) \frac{\partial \mathcal{T}_{i j}}{\partial q_{k}}= \\
& =\left(q_{1}+q_{2}+q_{3}\right) K_{\alpha}^{i j}-\sum_{k=1}^{3} q_{k}^{2}\left(q_{k}-1\right) \frac{\partial^{3} \mathcal{F}}{\partial q_{k} \partial a_{i} \partial a_{j}} .
\end{aligned}
$$

However, the SW differential (4.8) for the curve (5.5)

$$
d S=\frac{\sqrt{\alpha}\left(z-z_{0}\right)}{\sqrt{z(z-1)\left(z-q_{1}\right)\left(z-q_{2}\right)\left(z-q_{3}\right)}}=a_{1} d \omega_{1}+a_{2} d \omega_{2}
$$

states, that $\sqrt{\alpha}$ and $z_{0} \sqrt{\alpha}$ should be the linear functions of $a_{1}$ and $a_{2}$, which results in equations

$$
\operatorname{det} K_{\alpha}=0, \quad \operatorname{tr} K_{\alpha} K_{z}^{-1}=0
$$

for the (5.11). These equations, if considering them as constraints to the derivatives of the matrix elements of the period matrices $\partial_{k} \mathcal{T}_{\alpha \beta}$ should be considered independently of (5.6) (see appendix B). Generally, all such relations just follow from representation of

$$
x^{2}=\sum_{i=1}^{2 \tilde{g}-1} \frac{q_{i}\left(q_{i}-1\right)}{z(z-1)\left(z-q_{i}\right)} \frac{\partial \mathcal{F}}{\partial q_{i}} \underset{(4.13)}{=} \frac{1}{z(z-1) \prod_{j=1}^{2 \tilde{g}-1}\left(z-q_{j}\right)} \sum_{k=1}^{2 \tilde{g}-1} \mathcal{Q}_{\tilde{g}-1}^{2}\left(q_{k}\right) \prod_{i \neq k} \frac{z-q_{i}}{q_{k}-q_{i}}
$$

where the sum in the r.h.s. is just the Lagrange interpolation formula for the polynomial $\mathcal{Q}_{\tilde{g}-1}^{2}(z)$ with vanishing discriminant. 


\subsection{WDVV equations from residue formula}

Now let us show that prepotential of the $\mathrm{SU}(2)$ quiver gauge theories satisfies the WDVV equations $[26,27]$ as the function of full set of variables $\mathcal{F}=\mathcal{F}(\mathbf{a}, \mathbf{q})$. We have seen already, that in the case of $\mathrm{SU}(2)$ gauge quivers the residue formula descends to the base-curve $\Sigma_{0}$ of the SW curve $\Sigma$, and - adjusting to this case - we reformulate the statement of $[35,36]$ in the following way:

Theorem 4. Suppose that we have the formula

$$
\mathcal{F}_{I J K}=\sum_{f(z)=0} \operatorname{res} \frac{r_{I}(z) r_{J}(z) r_{K}(z)}{f(z)} R(z) d z
$$

where $f(z)$ is non-degenerate polynomial, $\operatorname{deg} f$ equals to the number of indices, $R(z)$ and $r_{I}(z)$ are holomorphic at zeroes of the polynomial. Then there is a relation [29] for the matrices $\left(\mathcal{F}_{I}\right)_{J K}=\mathcal{F}_{I J K}$

$$
\mathcal{F}_{I} \mathcal{F}_{J}^{-1} \mathcal{F}_{K}=\mathcal{F}_{K} \mathcal{F}_{J}^{-1} \mathcal{F}_{I}
$$

which is called the WDVV equation.

Proof. Define an auxiliary algebra $H_{S}$ of the functions on $N$ zeroes of $f(z)=\prod_{i=N}^{n}\left(z-\lambda_{i}\right)$ (which is obviously isomorphic to $\mathbb{C}^{N}$ ) by the relation

$$
\left(r_{I} \underset{S}{*} r_{J}\right)\left(\lambda_{i}\right)=S\left(\lambda_{i}\right) r_{I}\left(\lambda_{i}\right) r_{J}\left(\lambda_{i}\right)
$$

and the homomorphism $l_{S}: H_{S} \rightarrow \mathbb{C}$ by

$$
l_{S}(r)=\sum_{i=1}^{N} \frac{R\left(\lambda_{i}\right)}{f^{\prime}\left(\lambda_{i}\right) S\left(\lambda_{i}\right)^{2}} r\left(\lambda_{i}\right) .
$$

Compute the residue (5.15) directly, substituting $f(z)=\prod_{i=N}^{n}\left(z-\lambda_{i}\right)$

$$
\mathcal{F}_{I J K}=\sum_{i=1}^{N} \frac{R\left(\lambda_{i}\right)}{f^{\prime}\left(\lambda_{i}\right)} r_{I}\left(\lambda_{i}\right) r_{J}\left(\lambda_{i}\right) r_{K}\left(\lambda_{i}\right)=l_{S}\left(r_{I} \underset{S}{*} r_{J} \underset{S}{*} r_{K}\right) .
$$

Algebra $H_{S}$ is commutative and associative, and we can define it's structure constants

$$
r_{I} \underset{S}{*} r_{J}=\sum_{K} C_{I J}^{K} r_{K}
$$

or the operators of multiplication by $r_{I}$ as $\left(C_{I}\right)_{J}^{K}=C_{I J}^{K}$. Due to commutativity and associativity one has for these matrices

$$
C_{I} \cdot C_{J}=C_{J} \cdot C_{I} .
$$

Define now the bilinear form

$$
\eta_{I J}=l_{S}\left(r_{I} \underset{S}{*} r_{J}\right)
$$


which is obviously connected with $\left(\mathcal{F}_{I}\right)_{J K}=\mathcal{F}_{I J K}$ by

$$
\mathcal{F}_{I}=\eta \cdot C_{I} .
$$

Then

$$
\mathcal{F}_{I} \eta^{-1} \mathcal{F}_{K}=\mathcal{F}_{K} \eta^{-1} \mathcal{F}_{J}
$$

Using the possibility to choose arbitrarily the function $S(\lambda)$ one can adjust it to make $r_{J}=1$ for some fixed $J$ (we simply take $S\left(\lambda_{i}\right)=\frac{1}{r_{I}(\lambda)}$ and look at $(5.17)$ ), then $\eta=\mathcal{F}_{J}$, and (5.24) turns into (5.16).

Remark. Our algebra $H_{S}$ is isomorphic to the algebra of functions on $N$ points, so it obviously contains the unity operator. Namely, take the linear combination of the basis elements $e_{\alpha}=\sum_{I} \alpha_{I} r_{I}$ and then look at the multiplication by this element:

$$
\left(e_{\alpha}^{*} r_{J}\right)\left(\lambda_{i}\right)=\sum_{I} \alpha_{I} r_{I}\left(\lambda_{i}\right) S\left(\lambda_{i}\right) r_{J}\left(\lambda_{i}\right)=r_{J}\left(\lambda_{i}\right)
$$

To ensure the last equality, one has just to solve the system of $N$ linear equations:

$$
\frac{1}{S\left(\lambda_{i}\right)}=\sum_{I=1}^{N} \alpha_{I} r_{I}\left(\lambda_{i}\right), \quad \lambda_{i}=1, \ldots, N
$$

for $N$ variables $\alpha_{I}$, with the only requirement $\operatorname{det}_{I i}\left\|r_{I}\left(\lambda_{i}\right)\right\| \neq 0$. The corresponding

$$
\eta_{\alpha}=\sum_{I} \alpha_{I} \mathcal{F}_{I}
$$

is natural to consider as bilinear form, corresponding to the unity operator, but there is no claim that it does not depend on the dynamical variables.

\subsection{Proof for the quiver gauge theory}

As it was noticed, there are two important cases of the quiver gauge theories: ordinary and constrained one. All considerations will be very similar, so we introduce the following short-hand notation: $\left(z-v_{i}\right)^{(1 \mid 2)}$ which means that we should read $\left(z-v_{i}\right)^{1}$ in the ordinary case and $\left(z-v_{i}\right)^{2}$ in Zamolodchikov case.

Apply now residue formula (2.13) to the particular case of the massless $\mathrm{SU}(2)$ quiver gauge theory (2.21), rewriting it, first, for vanishing $\left\{\Delta_{i}\right\}$ in the form

$$
x^{2}=\frac{\alpha \prod_{s=1}^{\left(g-1 \mid \frac{g-1}{2}\right)}\left(z-v_{s}\left(q_{i}, a_{i}\right)\right)^{(1 \mid 2)}}{z(z-1) \prod_{k=1}^{g}\left(z-q_{i}\right)} .
$$

Express the corresponding $\mathbf{q}-$ and $\mathbf{a}$ - derivatives of $d S=x d z$ as:

$$
\begin{aligned}
& d \Omega_{k}=\frac{\partial}{\partial q_{k}} x d z=\left(\frac{1}{2} \frac{1}{z-q_{k}}+\sum_{s=1}^{\left(g-1 \mid \frac{g-1}{2}\right)} \frac{c_{s}}{z-v_{s}}+\frac{1}{2} \frac{\partial \log \alpha}{\partial q_{k}}\right) x d z=\mathfrak{R}_{k}(z) x d z \\
& d \omega_{i}=\frac{\partial}{\partial a_{i}} x d z=\left(\sum_{s=1}^{\left(g-1 \mid \frac{g-1}{2}\right)} \frac{b_{s}}{z-v_{s}}+\frac{1}{2} \frac{\partial \log \alpha}{\partial a_{i}}\right) x d z=\mathfrak{r}_{i}(z) x d z
\end{aligned}
$$


where $i=1, \ldots,\left(g \mid \frac{g+1}{2}\right), k=1, \ldots, g$, and unify now all variables into a single set $\left\{X_{I}\right\}=$ $\left\{a_{i}\right\} \cup\left\{q_{k}\right\},\left\{d \varpi_{I}\right\}=\left\{d \omega_{i}\right\} \cup\left\{d \Omega_{k}\right\},\left\{r_{I}\right\}=\left\{\mathfrak{r}_{i}\right\} \cup\left\{\mathfrak{R}_{k}\right\}$, with $I=1, \ldots,\left(2 g \mid \frac{3 g+1}{2}\right)$. The residue formula (2.13) gives rise to

$$
\frac{\partial^{3} \mathcal{F}}{\partial X_{I} \partial X_{J} \partial X_{K}}=2 \sum_{d x=0} \operatorname{res} \frac{d \varpi_{I} d \varpi_{J} d \varpi_{K}}{d x d z}=-2 \sum_{d z=0} \operatorname{res} \frac{\frac{\partial \log x}{\partial X_{I}} \frac{\partial \log x}{\partial X_{J}} \frac{\partial \log x}{\partial X_{K}}}{\frac{1}{x^{2}} \frac{d \log x}{d z}} d z
$$

where in the denominator of the r.h.s. we get explicitly

$$
\begin{aligned}
\frac{1}{x^{2}} \frac{d \log x}{d z} & =\frac{1}{2}\left(-\frac{1}{z}-\frac{1}{z-1}-\sum_{i=1}^{g} \frac{1}{z-q_{i}}+\sum_{s=1}^{\left(g-1 \mid \frac{g-1}{2}\right)} \frac{(1 \mid 2)}{z-v_{s}}\right) \frac{z(z-1) \prod_{i=1}^{g}\left(z-q_{i}\right)}{\alpha \prod_{s=1}^{\left(g-1 \mid \frac{g-1}{2}\right)}\left(z-v_{s}\right)^{(1 \mid 2)}}= \\
& =-\frac{2 f_{\left(2 g \mid \frac{3 g+1}{2}\right)}(z)}{\prod_{s=1}^{\left(g-1 \mid \frac{g-1}{2}\right)}\left(z-v_{s}\right)^{(2 \mid 3)}}
\end{aligned}
$$

where $f_{\left(2 g \mid \frac{3 g+1}{2}\right)}(z)$ is a polynomial of degree $\left(2 g \mid \frac{3 g+1}{2}\right)$. Therefore

$$
\frac{\partial^{3} \mathcal{F}}{\partial X_{I} \partial X_{J} \partial X_{K}}=\sum_{f_{(\bullet)}(z)=0} \operatorname{res} \frac{r_{I}(z) r_{J}(z) r_{K}(z)}{f_{\left(2 g \mid \frac{3 g+1}{2}\right)}(z)} \prod_{s=1}^{\left(g-1 \mid \frac{g-1}{2}\right)}\left(z-v_{s}\right)^{(2 \mid 3)} d z
$$

and since $r_{I}(z)$ are all holomorphic at zeroes of $f_{\left(2 g \mid \frac{3 g+1}{2}\right)}(z)$, and the total number of variables is $\left(2 g \mid \frac{3 g+1}{2}\right)$, one can immediately apply theorem 4 . It means, that we have proven, that the WDVV equations hold for the SW tau-function, as function of all periods and couplings, for both constrained and unconstrained case on equal footing.

\section{Conclusion}

In this paper we have studied in detail the properties of the $\mathrm{SU}(2)$ quiver gauge theories, along the lines proposed in [8]. We have derived and proved the residue formula for the third derivatives, and used it for some further applications.

We have shown, that the residue formula provides an effective way for the computation of the weak-coupling expansion of the quiver gauge theory prepotentials. These residue formulas can be used as a differential equation, which can be solved recursively, and this is equivalent to the expansion of the SW periods - the integrals of motion for these differential equations. Another application of the residue formula is that it leads immediately to the WDVV equations for the extended prepotential, once the number of critical points is equal to the number of deformations. We have checked above, that this condition holds both in the case of the full quiver theory, and its restricted or Zamolodchikov's case.

The Zamolodchikov case has attracted our special attention. We have completely described it in the SW approach for the quiver theory, showing that it arises after constraints, corresponding to arising of a massless state of a tri-fundamental matter. The prepotential then turn to be quadratic expressions in SW periods, forming a new class of conformal 
gauge theories, where the bare UV couplings are corrected only non-perturbatively. This renormalization can be described in terms of the Thomae formulas for the branching points of hyperelliptic curves, which generalize naturally the Zamolodchikov renormalization in the single $\mathrm{SU}(2)$ conformal theory.

For the higher rank gauge groups the situation seems to be far more complicated, but it looks like it can be studied by the methods, proposed in this paper. The extension to the higher-rank gauge theories can be possible started with extension of the Zamolodchikov's case, whose SW formulation is one of the main results of the present paper. Complete analysis of the higher rank case requires also the study of the higher Teichmüller spaces and corresponding deformations of the UV gauge theory, but the higher rank analogs of the Zamolodchikov case should be understandable in the SW terms, since on the CFT side it is described in terms of a systems of several scalar fields on Riemann surfaces. We plan to return to this problem elsewhere.

\section{A Conformal block in the Ashkin-Teller model}

Here we present the simplified derivation of the exact conformal block in $c=1$ AT model in terms convenient for the correspondence with the SW theory. The starting point in [18] is the operator algebra of the AT model which contains:

- the $\mathrm{U}(1)$-current $I(z)$, the Sugawara stress-energy tensor is $T(z)=: I(z)^{2}$ :;

- the Virasoro primary spin field $\sigma_{0}(z)$ and its first descendant in the current module $\sigma_{1}(z)$, which are $\mathbb{Z}_{2}$ twist-fields in terms of $I(z)$ (do not have the $\mathrm{U}(1)$ charge).

Consider the conformal or "current blocks" of the spin fields $\left\langle\sigma_{0}\left(z_{1}\right) \ldots \sigma_{0}\left(z_{n}\right)\right\rangle$, where the charges in the intermediate channel is fixed by

$$
\frac{1}{2 \pi i} \oint_{A_{\alpha}} I(z) d z=a_{\alpha}, \quad \alpha=1, \ldots, \tilde{g}=\frac{n}{2}-1
$$

where the $A$-cycles encircle each two spin fields, and are interpreted as canonical on the hyperelliptic curve

$$
y^{2}=\prod_{i=1}^{2 \tilde{g}+2}\left(z-z_{i}\right)
$$

introduced to make the correlator of spin-fields to be a single-valued function on this twofold cover of the initial sphere $\Sigma_{0}$. The operator product expansions [18]

$$
\begin{aligned}
& I(z) \sigma_{0}(0)=\frac{1}{2} z^{-\frac{1}{2}} \sigma_{1}(0)+\ldots \\
& I(z) \sigma_{1}(0)=\frac{1}{2} z^{-\frac{3}{2}} \sigma_{0}(0)+2 z^{-\frac{1}{2}} \partial \sigma_{0}(0)+\ldots
\end{aligned}
$$

predict for the ratios of the correlation functions

$$
F_{0}\left(z \mid\left\{z_{i}\right\}\right)=\frac{\left\langle I(z) \sigma_{0}\left(z_{1}\right) \ldots \sigma_{0}\left(z_{2 \tilde{g}+2}\right)\right\rangle}{\left\langle\sigma_{0}\left(z_{1}\right) \ldots \sigma_{0}\left(z_{2} \tilde{g}+2\right)\right\rangle}=\frac{\mathcal{Q}_{g-1}(z)}{y}
$$


where the r.h.s. is written from the (A.3) and contains a $g$-parametric polynomial, totally fixed by the period integrals (4.9), coming now from (A.1). For the ratio of slightly different correlation functions it follows from (A.3) and analytic properties

$$
F_{1}\left(z \mid\left\{z_{i}\right\}\right)=\frac{\left\langle I(z) \sigma_{1}\left(z_{1}\right) \sigma_{0}\left(z_{2}\right) \ldots \sigma_{0}\left(z_{2} \tilde{g}+2\right)\right\rangle}{\left\langle\sigma_{1}\left(z_{1}\right) \sigma_{0}\left(z_{2}\right) \ldots \sigma_{0}\left(z_{2} \tilde{g}+2\right)\right\rangle}=\frac{\mathcal{Q}_{g-1}(z)}{y}+\frac{d \Omega_{1}}{d z}
$$

where

$$
d \Omega_{1}=\frac{C_{1} d z}{y}\left(\frac{1}{z-z_{1}}+O_{g-1}(z)\right), \quad \oint_{A_{i}} d \Omega_{1}=0
$$

is the normalized Abelian differential on (A.2). The operator product expansion (A.3) inserted into (A.4) at $z \rightarrow z_{1}$ leads to relation

$$
\frac{\mathcal{Q}_{g-1}\left(z_{1}\right)}{\sqrt{\prod_{j \neq 1}\left(z_{1}-z_{j}\right)}}\left\langle\sigma_{0}\left(z_{1}\right) \sigma_{0}\left(z_{2}\right) \ldots \sigma_{0}\left(z_{2 \tilde{g}+2}\right)\right\rangle=\frac{1}{2}\left\langle\sigma_{1}\left(z_{1}\right) \sigma_{0}\left(z_{2}\right) \ldots \sigma_{0}\left(z_{2 \tilde{g}+2}\right)\right\rangle
$$

while inserted into (A.5) gives

$$
\frac{\left\langle\sigma_{0}\left(z_{1}\right) \sigma_{0}\left(z_{2}\right) \ldots \sigma_{0}\left(z_{2} \tilde{g}+2\right)\right\rangle}{\left\langle\sigma_{1}\left(z_{1}\right) \sigma_{0}\left(z_{2}\right) \ldots \sigma_{0}\left(z_{2} \tilde{g}+2\right)\right\rangle}=\frac{2 C_{1}}{\sqrt{\prod_{j \neq 1}\left(z_{1}-z_{j}\right)}}
$$

i.e. $4 C_{1} \mathcal{Q}_{g-1}\left(z_{1}\right)=\prod_{j \neq 1}\left(z_{1}-z_{j}\right)$, and

$$
2 \frac{\partial_{z_{1}}\left\langle\sigma_{0}\left(z_{1}\right) \sigma_{0}\left(z_{2}\right) \ldots \sigma_{0}\left(z_{2} \tilde{g}+2\right)\right\rangle}{\left\langle\sigma_{1}\left(z_{1}\right) \sigma_{0}\left(z_{2}\right) \ldots \sigma_{0}\left(z_{2} \tilde{g}+2\right)\right\rangle}=\frac{\mathcal{Q}_{g-1}\left(z_{1}\right)+C_{1} O_{g-1}\left(z_{1}\right)}{\sqrt{\prod_{j \neq 1}\left(z_{1}-z_{j}\right)}}-\frac{C_{1}}{2} \sum_{j \neq 1} \frac{1}{\left(z_{1}-z_{j}\right)^{3 / 2}} .
$$

These two formulas together result in

$$
\left(2 \partial_{z_{1}}-\frac{2 \mathcal{Q}_{g-1}^{2}\left(z_{1}\right)}{\prod_{j \neq 1}\left(z_{1}-z_{j}\right)}+\frac{1}{4} \sum_{j \neq 1} \frac{1}{z_{1}-z_{j}}+\frac{1}{2} O_{g-1}\left(z_{1}\right)\right)\left\langle\sigma_{0}\left(z_{1}\right) \sigma_{0}\left(z_{2}\right) \ldots \sigma_{0}\left(z_{2} \tilde{g}+2\right)\right\rangle=0 .
$$

Substituting the anzatz $\left\langle\sigma_{0}\left(z_{1}\right) \sigma_{0}\left(z_{2}\right) \ldots \sigma_{0}\left(z_{2} \tilde{g}+2\right)\right\rangle=e^{\mathcal{F}(\mathbf{a}, \mathbf{z})} \cdot G(\mathbf{z})$, where only the function in the exponent is a-dependent, one can extract from (A.10) the equality

$$
\frac{\partial \mathcal{F}}{\partial z_{i}}=\frac{\mathcal{Q}_{g-1}^{2}\left(z_{i}\right)}{\prod_{j \neq i}\left(z_{i}-z_{j}\right)}
$$

which coincides exactly with (4.13). It is also clear, that formulas (A.1), (4.9) turn into the first half of the periods of the SW differential. Hence, the exponential a-dependent contribution to the solution (A.10) can be obtained using the techniques presented in the main text of the paper, which leads immediately to the answer (4.3), where the quadratic form is already identified with the period matrix of (A.2), while it has been established only with some additional argumentation in the original paper [18]. 


\section{B More on derivatives of the period matrices}

Here we present some analysis of the equations (5.6) and (5.13), following from the Rauch formulas (5.1). Forgetting about the normalization conditions (4.10), one can consider equations (5.1) as parametrization of some submanifold in the space $\mathbb{A}^{\frac{1}{2} \tilde{g}(\tilde{g}+1)(2 \tilde{g}-1)}$ of the derivatives of our period matrix, where one can express the coordinates

$$
T_{\alpha \beta}^{k}=\prod_{l \neq k}\left(q_{k}-q_{l}\right) \frac{\partial \mathcal{T}_{\alpha \beta}}{\partial q_{k}}
$$

in terms of the $\tilde{g}^{2}$ coefficients $\left\{R_{\alpha \beta}\right\}$ of the polynomials $R_{\alpha}(z)$. From this point of view formulas (5.1) define the map $\mathbb{A}^{\tilde{g}^{2}} \mapsto \mathbb{A}^{\frac{1}{2} \tilde{g}(\tilde{g}+1)(2 \tilde{g}-1)}$ by quadratic functions

$$
T_{\alpha \beta}^{k}=R_{\alpha}\left(q_{k}\right) R_{\beta}\left(q_{k}\right)
$$

so it can be considered as the map $\mathbb{P}^{\tilde{g}^{2}-1} \mapsto \mathbb{P}^{\frac{1}{2} \tilde{g}(\tilde{g}+1)(2 \tilde{g}-1)-1}$.

The question, which allows to understand better the origin of equations (5.6), (5.13) is how to describe the image of this map. In the $\tilde{g}=2$ case, where we get $\mathbb{P}^{3} \mapsto \mathbb{P}^{8}$, the codimension is five and one needs at least five equations in $\mathbb{P}^{8}$. Notice, that we have already five independent equations in (5.6), but (5.13) give two extra.

Parameterizing our $\tilde{g}=2$ polynomials explicitly

$$
R_{1}(z)=a z+b, \quad R_{2}(z)=c z+d
$$

where $(a: b: c: d)$ are the homogeneous coordinates on $\mathbb{P}^{3}$, one can write

$$
T_{11}^{i}=\left(a q_{i}+b\right)^{2}, \quad T_{12}^{i}=\left(a q_{i}+b\right)\left(c q_{i}+d\right), \quad T_{22}^{i}=\left(c q_{i}+d\right)^{2}, \quad i=1,2,3 .
$$

Since all equations are quadratic in $a, b, c, d$ the intersection with the general codimension-3 plane contains $2^{3}=8$ points, so the degree of the image is 8 .

Suppose now, that the image is a total intersection in $\mathbb{P}^{8}$, then it should be determined by five polynomials $P_{d_{1}}, \ldots, P_{d_{5}}$, such that $d_{1} \cdot \ldots \cdot d_{5}=8$. It is possible only if at least two powers are $d_{i}=1$, therefore the image lies in a hyperplane. However, this turns to be impossible, since in such case one gets a linear equation

$$
\sum_{\alpha, \beta, k} C_{\alpha \beta}^{k} T_{\alpha \beta}^{k}=0
$$

which is immediately rewritten as

$$
C_{a a} a^{2}+C_{a b} a b+\ldots+C_{d d} d^{2}=0
$$

true $\forall(a, b, c, d)$, but this is impossible if $C_{I J} \neq 0$. Hence, we come to a contradiction, and our surface does not lie in the hyperplane. In particular, it means, that the equations (5.13) are not the consequence of (5.6), and should be considered independently. 


\section{Acknowledgments}

We are grateful to I. Krichever, A. Morozov and T. Shabalin for useful discussions, and to N. Nekrasov and V. Pestun for their comments of related topics and critical remarks. The preliminary results have been reported at the Russian-Japanese JSPS/RFBR workshop on Integrability and Gauge/String Duality in Moscow and at the workshop on Integrability and Gauge Theory, held within the program "Cohomology in Mathematics and Physics" of the Euler Institute in St.-Petersburg in September-October 2013.

The work was carried out within the research grant 13-05-0006 of the NRU HSE Academic Program support in 2013, and joint Ukrainian-Russian (NAN-RFBR) project 01-01-14 (P.G.) and 14-01-90404 (A.M.). The work of P.G. has been also supported by the grant of Laboratory of the Algebraic Geometry (HSE). The work of A.M. has been also supported by RFBR grant 14-01-00547, by joint RFBR/JSPS project 12-02-92108, by the Program of Support of Scientific Schools (NSh-1500.2014.2), and by the Russian Ministry of Education.

Open Access. This article is distributed under the terms of the Creative Commons Attribution License (CC-BY 4.0), which permits any use, distribution and reproduction in any medium, provided the original author(s) and source are credited.

\section{References}

[1] D. Gaiotto, N=2 dualities, JHEP 08 (2012) 034 [arXiv:0904.2715] [INSPIRE].

[2] N. Seiberg and E. Witten, Electric-magnetic duality, monopole condensation and confinement in $N=2$ supersymmetric Yang-Mills theory, Nucl. Phys. B 426 (1994) 19 [Erratum ibid. B 430 (1994) 485-486] [hep-th/9407087] [INSPIRE].

[3] A.S. Losev, A. Marshakov and N.A. Nekrasov, Small instantons, little strings and free fermions, in From fields to strings: circumnavigating theoretical physics. Vol. 1, Ian Kogan Memorial Collection, pg. 581-621, hep-th/0302191 [INSPIRE].

[4] N. Nekrasov and A. Okounkov, Seiberg-Witten theory and random partitions, hep-th/0306238 [INSPIRE].

[5] A. Braverman, Instanton counting via affine Lie algebras. 1. Equivariant J functions of (affine) flag manifolds and Whittaker vectors, math/0401409 [INSPIRE].

[6] A. Braverman and P. Etingof, Instanton counting via affine Lie algebras II: From Whittaker vectors to the Seiberg-Witten prepotential, math/0409441 [INSPIRE].

[7] L.F. Alday, D. Gaiotto and Y. Tachikawa, Liouville Correlation Functions from Four-dimensional Gauge Theories, Lett. Math. Phys. 91 (2010) 167 [arXiv:0906.3219] [INSPIRE].

[8] A. Marshakov, Tau-functions for Quiver Gauge Theories, JHEP 07 (2013) 068 [arXiv: 1303.0753] [INSPIRE].

[9] I.M. Krichever, The tau function of the universal Whitham hierarchy, matrix models and topological field theories, Commun. Pure Appl. Math. 47 (1994) 437 [hep-th/9205110] [INSPIRE]. 
[10] A. Gorsky, I. Krichever, A. Marshakov, A. Mironov and A. Morozov, Integrability and Seiberg-Witten exact solution, Phys. Lett. B 355 (1995) 466 [hep-th/9505035] [INSPIRE].

[11] A. Losev, N. Nekrasov and S.L. Shatashvili, Issues in topological gauge theory, Nucl. Phys. B 534 (1998) 549 [hep-th/9711108] [inSPIRE].

[12] A. Gorsky, A. Marshakov, A. Mironov and A. Morozov, RG equations from Whitham hierarchy, Nucl. Phys. B 527 (1998) 690 [hep-th/9802007] [INSPIRE].

[13] A. Marshakov and N. Nekrasov, Extended Seiberg-Witten Theory and Integrable Hierarchy, JHEP 01 (2007) 104 [hep-th/0612019] [INSPIRE].

[14] A. Marshakov, On Microscopic Origin of Integrability in Seiberg-Witten Theory, Theor. Math. Phys. 154 (2008) 362 [arXiv:0706.2857] [InSPIRE].

[15] N.A. Nekrasov, Seiberg-Witten prepotential from instanton counting, Adv. Theor. Math. Phys. 7 (2004) 831 [hep-th/0206161] [INSPIRE].

[16] N. Nekrasov and V. Pestun, Seiberg-Witten geometry of four dimensional $N=2$ quiver gauge theories, arXiv:1211.2240 [INSPIRE].

[17] L. Hollands, C.A. Keller and J. Song, Towards a $4 d / 2 d$ correspondence for Sicilian quivers, JHEP 10 (2011) 100 [arXiv:1107.0973] [INSPIRE].

[18] A.B. Zamolodchikov, Conformal Scalar Field on the Hyperelliptic Curve and Critical Ashkin-teller Multipoint Correlation Functions, Nucl. Phys. B 285 (1987) 481 [INSPIRE].

[19] A.B. Zamolodchikov, Conformal symmetry in two-dimensions: an explicit recurrence formula for the conformal partial wave amplitude, Commun. Math. Phys. 96 (1984) 419 [INSPIRE].

[20] A.B. Zamolodchikov, Two-dimensional conformal symmetry and critical four-spin correlation functions in the Ashkin-Teller model, Sov. Phys. JETP 63 (1986) 1061.

[21] A.B. Zamolodchikov, Conformal symmetry in two-dimensional space: Recursion representation of conformal block, Theor. Math. Phys. 73 (1987) 1088.

[22] T.W. Grimm, A. Klemm, M. Mariño and M. Weiss, Direct Integration of the Topological String, JHEP 08 (2007) 058 [hep-th/0702187] [inSPIRE].

[23] A. Marshakov, A. Mironov and A. Morozov, Zamolodchikov asymptotic formula and instanton expansion in $N=2$ SUSY $N_{f}=2 N_{c}$ QCD, JHEP 11 (2009) 048 [arXiv:0909.3338] [INSPIRE].

[24] R. Poghossian, Recursion relations in CFT and N=2 SYM theory, JHEP 12 (2009) 038 [arXiv:0909.3412] [INSPIRE].

[25] T. Eguchi and K. Maruyoshi, Penner Type Matrix Model and Seiberg-Witten Theory, JHEP 02 (2010) 022 [arXiv:0911.4797] [INSPIRE].

[26] E. Witten, On the Structure of the Topological Phase of Two-dimensional Gravity, Nucl. Phys. B 340 (1990) 281 [inSPIRE].

[27] R. Dijkgraaf, H.L. Verlinde and E.P. Verlinde, Topological strings in $d<1$, Nucl. Phys. B 352 (1991) 59 [INSPIRE].

[28] B. Dubrovin, Geometry of $2-D$ topological field theories, hep-th/9407018 [INSPIRE].

[29] A. Marshakov, A. Mironov and A. Morozov, WDVV-like equations in $N=2$ SUSY Yang-Mills theory, Phys. Lett. B 389 (1996) 43 [hep-th/9607109] [INSPIRE]. 
[30] L. Chekhov, A. Marshakov, A. Mironov and D. Vasiliev, $D V$ and $W D V V$, Phys. Lett. B 562 (2003) 323 [hep-th/0301071] [INSPIRE].

[31] A. Marshakov and A. Mironov, $5-D$ and $6-D$ supersymmetric gauge theories: Prepotentials from integrable systems, Nucl. Phys. B 518 (1998) 59 [hep-th/9711156] [INSPIRE].

[32] M. Matone, Modular invariance and structure of the exact Wilsonian action of $N=2$ supersymmetric Yang-Mills theory, Phys. Rev. Lett. 78 (1997) 1412 [hep-th/9610204] [INSPIRE].

[33] A. Litvinov, S. Lukyanov, N. Nekrasov and A. Zamolodchikov, Classical Conformal Blocks and Painleve VI, arXiv:1309.4700 [inSPIRE].

[34] E. D'Hoker, I.M. Krichever and D.H. Phong, The effective prepotential of $N=2$ supersymmetric $\mathrm{SU}(N(c))$ gauge theories, Nucl. Phys. B 489 (1997) 179 [hep-th/9609041] [INSPIRE].

[35] A. Marshakov, Associativity equations in effective SUSY quantum field theories, hep-th/0108023 [INSPIRE].

[36] A. Marshakov, On associativity equations, Theor. Math. Phys. 132 (2002) 895 [hep-th/0201267] [INSPIRE].

[37] M. Sato, T. Miwa and M. Jimbo, Holonomic quantum fields I, Publ. RIMS 14 (1978) 223.

[38] M. Sato, T. Miwa and M. Jimbo, Holonomic quantum fields II - The Riemann-Hilbert Problem, Publ. RIMS 15 (1979) 201.

[39] M. Sato, T. Miwa and M. Jimbo, Holonomic quantum fields III, Publ. RIMS 15 (1979) 577.

[40] M. Sato, T. Miwa and M. Jimbo, Holonomic quantum fields IV, Publ. RIMS 15 (1979) 871.

[41] M. Sato, T. Miwa and M. Jimbo, Holonomic quantum fields V, Publ. RIMS 16 (1980) 531.

[42] O. Gamayun, N. Iorgov and O. Lisovyy, Conformal field theory of Painlevé VI, JHEP 10 (2012) 038 [Erratum ibid. 1210 (2012) 183] [arXiv:1207.0787] [INSPIRE].

[43] A. Kitaev and D. Korotkin, On solutions of the Schlesinger Equations in Terms of $\Theta$-Functions, Int. Math. Res. Not. 17 (1998) 877 [math-ph/9810007] [INSPIRE].

[44] A.A. Belavin, M.A. Bershtein, B.L. Feigin, A.V. Litvinov and G.M. Tarnopolsky, Instanton moduli spaces and bases in coset conformal field theory, Comm. Math. Phys. 319 (2013) 269 [arXiv: 1111.2803] [INSPIRE].

[45] J.Fay, Theta-functions on Riemann surfaces, Springer, Lect. Notes Math. 352 (1973).

[46] D.Mumford, Tata Lectures on Theta, Birkhäuser, (1988).

[47] V. Knizhnik, Multiloop amplitudes in the theory of quantum strings and complex geometry, Sov. Phys. Usp. 32 (1989) 945 [Usp. Fiz. Nauk 159 (1989) 401] [InSPIRE].

[48] D. Lebedev and A. Morozov, Statistical Sums of Strings on Hyperelliptic Surfaces, Nucl. Phys. B 302 (1988) 163 [inSPIRE]. 